LMU-ASC 1/05

MPP-2005-3

hep-th/0501139

\title{
F-theory Flux, Destabilization of Orientifolds and Soft Terms on D7-Branes
}

\author{
D. Lüst, ${ }^{a, b}$ P. Mayr, ${ }^{a}$ S. Reffert, ${ }^{b}$ and S. Stieberger ${ }^{a}$ \\ ${ }^{a}$ Arnold-Sommerfeld-Center for Theortical Physics, \\ Department für Physik, Ludwig-Maximilians-Universität München, \\ Theresienstraße 37, D-80333 München, Germany \\ ${ }^{b}$ Max-Planck-Institut für Physik, Föhringer Ring 6, D-80805 München, Germany
}

\begin{abstract}
We use F-theory to derive a general expression for the flux potential of type II compactifications with D7/D3 branes, including open string moduli and 2-form fluxes on the branes. Our main example is F-theory on $\mathrm{K} 3 \times \mathrm{K} 3$ and its orientifold limit $T^{2} / \mathbf{Z}_{2} \times \mathrm{K} 3$. The full scalar potential cannot be derived from the bulk superpotential $W=\int \Omega \wedge G_{3}$ and generically destabilizes the orientifold. Generically all open and closed string moduli are fixed, except for a volume factor. An alternative formulation of the problem in terms of the effective supergravity is given and we construct an explicit map between the F-theory fluxes and gaugings. We use the superpotential to compute the effective action for flux compactifications on orbifolds, including the $\mu$-term and soft-breaking terms on the D7-brane world-volume.
\end{abstract}

January 2005 


\section{Introduction}

Type II compactifications with fluxes and D7/D3 brane systems play a prominent role in the search for semi-realistic 4-dimensional string vacua.The effect of 3 -form background fluxes has been described by a superpotential [1] [2] [3]

$$
W=\int \Omega \wedge G_{3},
$$

where $\Omega$ is the holomorphic $(3,0)$-form on the compactification manifold $Y$ and $G_{3}=F_{3}-S_{3}$ the quantized background flux. In the latter expression, $F_{3}\left(H_{3}\right)$ denotes the flux in the RR (NS) sector and $S$ is the complex type IIB dilaton.

Formally, the above expression for the superpotential is identical to that for closed string compactifications without D-branes on a Calabi-Yau 3-fold $Y$. However, in the latter case, the periods $\int \Omega$ of the holomorphic 3 -form $\Omega$ compute the exact scalar potential [4] [5], whereas the above formula for the orientifold relies on a supergravity approximation.

In the following we improve on previous results by computing the flux superpotential for certain D7/D3 orientifolds directly from F-theory [6]. The set of available fluxes in the F-theory is considerably larger then those described by the bulk potential, and includes 2-form fluxes on the D7-branes. The vacuum structure of the potential obtained from F-theory differs in various respects from studies based on eq.(11.1), most notably

- For general fluxes all moduli are stabilized, except for a single volume factor. This includes the dilaton, Kähler and complex structure moduli as well as D7-brane moduli.

- The general flux potential destabilizes the orientifold limit and drives the theory into a regime with varying dilaton, where the orientifold approximation is not valid and F-theory is needed.

- The supersymmetric flux can not be assigned Hodge type $(2,1)$ and there is no strict relation between $(2,1)$ fluxes and mass terms for D7-brane moduli.

The flux compactification of F-theory can be rephrased also in terms of an effective gauged supergravity, and we give an explicit dictionary between the fluxes and certain gaugings. An interesting aspect of the derivation is that the F-theory geometry provides important physical information beyond the classical effective supergravity, such as the integral lattice of charged BPS states and the quantization conditions on the gaugings. 
We then turn to a detailed discussion of the case of the $\mathrm{K} 3$ orbifold $T^{4} / \mathbf{Z}_{2}$, which arises also as the $\mathcal{N}=2$ supersymmetric sector of $\mathcal{N}=1$ orbifolds and plays a prominent role in the study of intersecting brane worlds. In this orbifold limit we study further the gaugings in the supergravity approximation and the flux dependent superpotential. We present explicitly its dependence on the dilaton, complex structure moduli and open string moduli and the dictionary between the string theory fluxes and the supergravity gaugings. In particular, the superpotential, now also depending on the $D 7$-brane moduli, gives rise to a $\mu$-term.

These results allow to study the $D=4$ effective $\mathcal{N}=1$ supergravity action with spontaneous supersymmetry breaking due to non-vanishing auxiliary $F$-term components of the moduli fields. In this framework we calculate the flux-induced soft supersymmetry breaking terms of the effective four-dimensional $D 7$-brane gauge theory. In lines of Giudice-Masiero, the $\mu$-term comprises together with supersymmetry breaking terms into an effective supersymmetric mass term, which gives masses to the non-chiral fermions of the D7-brane moduli superfields. The latter depends on $(2,1)$-form fluxes only in the case of vanishing world-volume 2-form fluxes on the $D 7$-branes, but receives a $(1,2)$-dependent piece in the presence of fluxes. On the other hand, the scalar fields of $D 7$-brane moduli, describing their positions, receive both 3 - and 2-from flux dependent contributions.

The organization of this note is as follows. In sect. 2 we compute the general potential in F-theory on $\mathrm{K} 3 \times \mathrm{K} 3$ for bulk and brane fluxes and describe its orientifold and orbifold limits. In sect. 3 we study the general vacuum structure of the potential and discuss some of the new features in a few examples. In sect. 4 we describe the F-theory potentials in terms of an effective gauged supergravity and give an explicit dictionary between the 3 -form and 2-form fluxes in F-theory and gaugings in supergravity. In sect. 5 we study in detail the orbifold $T^{4} / \mathbf{Z}_{2}$ and derive its supergravity description in the presence of bulk 3 -form and world volume 2 -form fluxes on the $D 7$-branes. In sect. 6 we compute the $\mu$-term as well as the soft breaking masses on the $D 7$-brane arising from the flux potential. Some necessary details on the K3 manifold and its orientifold and orbifold limits are discussed in the appendix.

\section{Flux potentials in $M$ - and $F$-theory}

To compute flux potentials in F-theory, we can build on the work on Mtheory compactifications on Calabi-Yau 4-folds $X_{4}$ in [7] [1] [2] [8]. If $X_{4}$ is elliptically fibered, M-theory on $X_{4}$ is the same as F-theory on $X_{4} \times S^{1}$ [9]. The 4-dimensional 
F-theory compactification on $X_{4}$ arises in the limit of infinite radius of $S^{1}$. Some aspects of the F-theory limit have been discussed already in [1] [2] [10].

In the following we will mostly consider the case $X_{4}=\mathrm{K} 3 \times \mathrm{K} 3$. This compactification reduces to the type IIB orientifold on $T^{2} / \mathbf{Z}_{2} \times \mathrm{K} 3$ in a particular limit [11]. We will discuss this orientifold limit later in some detail. Instead of a product $\mathrm{K} 3 \times \mathrm{K} 3$ one could also consider a non-trivial fibration of $\mathrm{K} 3$ over a 2 complex dimensional base. Some of the following arguments could be generalized to this case.

In the 4-dimensional limit of $\mathrm{F}$-theory on $X_{4}$, the two $\mathrm{K} 3$ factors play very different roles. The first K3 factor, on which F-theory is compactified to 8 dimensions has to be elliptically fibered [6] and will be denoted by $\widetilde{X}_{V}$. The second factor for the compactification from 8 to 4 dimensions will be denoted by $X_{H}$. The notation reflects an important distinction of how the moduli of the two K3 factors enter in the effective 4-dimensional theory. The latter is a standard effective $\mathcal{N}=2$ supergravity with a number $n_{V}$ of vector multiplets and a number $n_{H}$ of hyper multiplets. Up to one exception, the moduli of the elliptic F-theory manifold $\widetilde{X}_{V}$ end up in vector multiplets, whereas the moduli of $X_{H}$ end up in the hypers. ${ }^{-}$The flux potential will reflect this distinction in a natural way.

\subsection{Superpotentials in M-theory}

Upon $S^{1}$ compactification, F-theory on the elliptically fibered 4 -fold $X_{4}$ is equivalent to M-theory with an elliptic fiber of volume $k_{E}$ inverse proportional to the radius of $S^{1}$. In M-theory, internal flux of the 4 -form field strength $G_{4}$ induces two different superpotentials [1] [8]:

$$
W=\int_{X_{4}} \Omega \wedge G_{4}, \quad \breve{W}=\int_{X_{4}} J \wedge J \wedge G_{4},
$$

where $J$ is the Kähler form and $\Omega$ the holomorphic $(4,0)$-form on $X_{4}$. By definition, the superpotentials $W$ and $\breve{W}$ depend on complex structure and Kähler moduli of $X_{4}$, respectively.

Consider an integral 4-form $G_{4}$ that is the sum of a product of integral 2-forms on the two K3 factors

$$
G_{4}=\sum \underline{\mathrm{G}}^{I \Lambda} \eta_{I} \wedge \widetilde{\eta}_{\Lambda}, \quad \underline{\mathrm{G}}^{I \Lambda} \in \mathbf{Z} .
$$

1 The effective supergravity is discussed in some detail in sect. 4. 
Here $\eta_{I}$ denotes a basis of $H^{2}\left(X_{H}, \mathbf{Z}\right)$ and similar quantities with a tilde are used for the same objects on the elliptic K3, $\widetilde{X}_{V}$. See app. A for some properties of the $\mathrm{K} 3$ cohomology and notations. The integral matrix $\underline{\mathrm{G}}^{I \Lambda}$ characterizes the flux on the two K3 factors.

On $X_{4}=\mathrm{K} 3 \times \mathrm{K} 3$, the 4 -form $\Omega$ and the Kähler form $J$ decompose as

$$
\Omega=\omega \wedge \widetilde{\omega}, \quad J=j+\widetilde{j},
$$

where $\omega$ and $j$ are the holomorphic 2-form and Kähler form on $X_{H}$ and similarly for the tilded quantities on $\widetilde{X}_{V}$. In fact, writing

$$
\omega=\omega^{1}+i \omega^{2}, \quad j=\omega^{3}
$$

the three 2-forms $\omega^{i}$ transform as a triplet under the trivial $S U(2)$ part of the holonomy of K3. Choosing a basis of cycles $\gamma_{I} \in H_{2}(K 3, \mathbf{Z})$ dual to $\left\{\eta_{I}\right\}$, one has the triplet of period vectors

$$
\Pi^{I, x}=\int_{\gamma_{I}} \omega^{x}=\int \omega^{x} \wedge \eta^{I}=\int \omega^{x} \wedge \eta_{J} M^{J I}
$$

These will be the central objects in the description of the full moduli dependence of the potential in the following. The indices $I$ are raised an lowered with the help of the intersection matrix $M_{I J}$ defined in (A.1). We use also

$$
\Pi^{I}=\Pi^{I, 1}+i \Pi^{I, 2}
$$

to denote the complex combination corresponding to the holomorphic 2-form. Similar notations will be used for the "upper" $\mathrm{K} 3, \widetilde{X}_{V}$.

Note that the definition of the superpotential (2.1) seems to select a preferred direction in $S U(2)$ corresponding to the Kähler form $j$. This defines a fixed complex structure on $X_{H}$, as well as on the moduli space. This will be important later when comparing with the effective $\mathcal{N}=1$ supergravity.

With the above notations, the superpotentials become

$$
W=\sum_{I \Lambda} \underline{\mathrm{G}}_{I \Lambda} \Pi^{I} \widetilde{\Pi}^{\Lambda}, \quad \quad \breve{W}=\sum_{I \Lambda} \underline{\mathrm{G}}_{I \Lambda} \Pi^{I, 3} \widetilde{\Pi}^{\Lambda, 3} .
$$

Thus the potential is simply a homogeneous polynomial of degree $(1,1)$ in the periods of the two K3 factors. One can now evaluate the moduli dependence of the potential by computing the period integrals. An important property of the K3 periods is 
that they may serve locally as good projective coordinates on the moduli space, except for certain quadratic constraints. It follows that the potentials are also inhomogeneous polynomials of degree $(2,2)$ in the moduli. We will describe an appropriate parametrization of the periods momentarily.

Finally, the contribution of $G_{4}$ to the membrane tadpole [12] is

$$
N_{F}=\frac{1}{2} \int G_{4} \wedge G_{4}=\frac{1}{2} \underline{\mathrm{G}}^{I} \Lambda \underline{\mathrm{G}}_{I}^{\Lambda} .
$$

\subsection{Superpotentials in F-theory}

Under certain conditions, the potentials (2.5) lift to flux potentials of the Ftheory compactification to four dimensions. It is useful to keep in mind two aspects that are new in this 4-dimensional F-theory limit, which distinguishes the two K3 factors. As mentioned already, the periods of the "upper" $\mathrm{K} 3, \widetilde{X}_{V}$, become related to vector multiplets, whereas the periods of the "lower" $\mathrm{K} 3, X_{H}$, are related to hyper multiplets,

$$
\Pi^{I} \longleftrightarrow 4 \mathrm{~d} \text { hypers, } \quad \widetilde{\Pi}^{\Lambda} \longleftrightarrow 4 \mathrm{~d} \text { vectors. }
$$

Secondly, as further discussed in sect. 3, the superpotential $W$ can be identified with an $\mathcal{N}=1 F$-term under certain conditions, while $\breve{W}$ becomes related to $D$-terms.

The conditions that the M-theory flux compactification has an 4d F-theory limit are as follows. To lift the 3-dimensional M-theory to F-theory in 4 dimensions, $\widetilde{X}_{V}$ must be elliptically fibered. The dual $E$ of the class of the fiber and the dual $B$ of the class of the $\mathbf{P}^{1}$ base of the fibration define two elements of the so-called Picard group

$$
\operatorname{Pic}\left(\widetilde{X}_{V}\right)=H^{1,1}\left(\widetilde{X}_{V}\right) \cap H^{2}(X, \mathbf{Z}),
$$

with intersections

$$
\int E \wedge E=2, \quad \int E \wedge B=1, \quad \int B \wedge B=0 .
$$

It has been argued in [1] [2], that a selfdual 4-form flux on $X_{4}$ must be of the form (2.2) and in addition orthogonal to the 2-plane $U$ spanned by $(E, B)$. Since $\operatorname{dim} H^{2}\left(\widetilde{X}_{V}\right)=22$, the forms $\widetilde{\eta}_{\Lambda}$ appearing in the decomposition (2.2) are restricted to the 20-dimensional space $\Gamma_{V} \subset H^{2}\left(\widetilde{X}_{V}, \mathbf{Z}\right)$ orthogonal to $U$. The dimension 20 of this space is precisely the number of $U(1)$ gauge fields in the 8-dimensional compactification of F-theory on $\widetilde{X}_{V}$. One may naturally think of the flux matrix $\underline{\mathrm{G}}^{I \Lambda}$ as describing the 2-form fluxes $\mathcal{F}^{\Lambda}$ of $20 U(1)$ gauge fields on the "lower" K3 $X_{H}$. 
Fixing the radius of $S^{1}$

Note that the radius of $S^{1}$ in the compactification from 4 to 3 dimensions is a modulus in the theory without flux. If one searches for $4 \mathrm{~d}$ flux vacua with a maximal number of moduli fixed, one would like to fix also the radius of $S^{1}$ at infinity. In fact there is another choice of flux in addition to the one discussed above, which is appropriate. One can check from (2.6) that a flux component

$$
G_{4}^{(4 d)} \sim \eta_{I} \wedge B
$$

with $\eta_{I}$ a primitive 2-form on $X_{H}$, is primitive in the F-theory limit.2 Switching on a component (2.7) induces a term in $\breve{W}$ that drives the $3 \mathrm{~d}$ M-theory to $4 \mathrm{~d}$ F-theory.

\subsection{Moduli dependence of $W$}

As discussed already the, perhaps somewhat abstract looking, formulae (2.5) contain already the full moduli dependence of the four-dimensional potential. We refer again to the app. A for a detailed discussion of the parametrization of the K3 periods. Only for simplicity of exposition we assume that the "lower" K3 is also elliptically fibered. In a certain parametrization, the holomorphic periods $\Pi$ and $\widetilde{\Pi}$ take the form (A.7)

$$
\Pi^{I}=\left(\begin{array}{c}
1 \\
-U_{1} U_{2}+\frac{1}{2} W^{a} W^{a} \\
U_{1} \\
U_{2} \\
W^{a}
\end{array}\right), \quad \widetilde{\Pi}^{\Lambda}=\left(\begin{array}{c}
1 \\
-S U+\frac{1}{2} C^{a} C^{a} \\
S \\
U \\
C^{a}
\end{array}\right)
$$

where $a=1, \ldots 16$.

The 18 complex scalars $\left(U_{i}, W^{a}\right)$ are members of $4 \mathrm{~d}$ (hyper) multiplets that parametrize the complex structure of the "lower" $\mathrm{K} 3, X_{H}$. For $W^{i}=0$, the period vector $\Pi^{I}$ may describe the $\mathrm{K} 3$ orbifold $T^{2} \times T^{2} / \mathbf{Z}_{2}$, with $U_{i}$ the two complex structure parameters of the two $T^{2}$ 's. On the other hand, the $W^{i}$ describe the deformations away from the orbifold.

The 18 complex scalars $\left(S, U, C^{a}\right)$ are components of $4 \mathrm{~d}$ vector multiplets. Let us compare this in direction to the spectrum of the type II orientifold $T^{2} / \mathbf{Z}_{2} \times$ K3, where the scalars in the vector multiplets include the dilaton $S^{\prime}$, the complex structure $U^{\prime}$ of $T^{2}$ and 16 D7-brane positions $C^{\prime a}$. Here we use primes to distinguish the orientifold fields from the moduli appearing in the periods $\widetilde{\Pi}^{\Lambda}$. One is tempted

2 Recall that a primitive 4 -form $G_{4}$ fulfills $J \wedge G_{4}$ and minimizes the potential $\breve{W}$ [迻]. 
to identify these two sets of scalars and identify the orientifold limit with $C^{a}=0$ in (2.8). However this identification is non-trivial as illustrated in app. B; the result of the computation is that the naive identification of the primed and unprimed field can be justified, but the integrality properties are obscured in such a parametrization. In other words, the "period vector" $\Pi^{I}$, with $C^{a}=0$ corresponding to the orientifold, is not defined w.r.t. a basis that generates $H_{2}\left(\widetilde{X}_{V}, \mathbf{Z}\right)$ over the integers. Since the integrality properties can nevertheless be recovered from the known transformation to the integral basis, we neglect this issue for the moment and take it as granted that $C^{a}=0$ describes the orientifold limit, and moreover $S$ and $U$ can be identified with the dilaton and complex structure of $T^{2}$, respectively.

Using the parametrization (2.8), the general formula for the F-theory superpotential (2.5) becomes

$$
\begin{aligned}
W & =p_{1}+p_{2}\left(\frac{1}{2} C^{a} C^{a}-U S\right)+p_{3} S+p_{4} U+\sum_{a=1}^{16} p_{4+a} C^{a} \\
& =\int_{X_{H}} \omega \wedge g_{2,0},
\end{aligned}
$$

where

$$
g_{2,0}=\eta_{I} \underline{\mathrm{G}}_{\Lambda}^{I} \widetilde{\Pi}^{\Lambda}, \quad p_{\Lambda}=\Pi^{I} \underline{\mathrm{G}}_{I \Lambda} .
$$

The notation anticipates that $g_{2,0} \in H^{2}\left(X_{H}\right)$ will be of type $(2,0)$ at the minimum, as will be discussed later. Both forms of $W$ will be useful when studying the vacuum configurations.

\subsection{Moduli dependence of $\breve{W}$}

The superpotential for the Kähler moduli is (2.5)

$$
\breve{W}=\left(\int_{X_{H}} j \wedge \eta^{I}\right) \underline{\mathrm{G}}_{I \Lambda}\left(\int_{\widetilde{X}_{V}} \widetilde{j} \wedge \widetilde{\eta}^{\Lambda}\right) .
$$

In the F-theory limit, the Kähler form on $\widetilde{X}_{V}$ is proportional to the volume form on the base $\mathbf{P}^{1}$ of the elliptic fibration. It follows that the integrals on $\tilde{X}_{V}$ in the above formula are automatically zero; however the derivatives $\frac{\partial}{\partial \xi_{\Lambda}} \Pi^{\Sigma, 3}$ with respect to the Kähler volumes $\xi_{\Lambda} \rightarrow 0$ are not. With an appropriate choice of coordinates, the content of the variational equations orthogonal to the overall volume is

$$
\partial_{\xi_{\Lambda}} \breve{W}=\int_{X_{H}} j \wedge \eta^{I} \underline{\mathrm{G}}_{I \Lambda}=\Pi^{I, 3}\left(T^{i}\right) \underline{\mathrm{G}}_{I \Lambda}=0
$$

As will be further discussed below, these equations will correspond to $D$-term equations for differences of $U(1)$ factors from the D7-branes, that restrict the Kähler moduli $T^{i}$ of $X_{H}$. Note that $D$ - and $F$-terms cannot be chosen independently, since both of them are defined by the same flux matrix $\underline{\mathrm{G}}_{I J}$. 


\subsection{Orientifold limit and bulk moduli}

There is a large amount of literature on flux potentials of type IIB orientifolds, based on the study of the bulk superpotential eq.(11.1). To relate these results to the present F-theory picture, it is instructive to study in some detail the orientifold limit.

The bulk fields of the 8-dimensional type II compactification on the orientifold $T^{2} / \mathbf{Z}_{2}$ comprise two complex moduli, the complex type IIB dilaton $S$ and the complex structure modulus $U$ of $T^{2}$, as well as one real scalar $\alpha$ parametrizing the volume of $T^{2}$. In the K3 moduli space, the orientifold limit can be described by the following Weierstrass form for the elliptic fibration [11]

$$
y^{2}+x^{3}+x \widehat{z}^{4} f+\widehat{z}^{6} g, \quad f=a \prod_{i=1}^{4}\left(z-z_{i}\right)^{2}, \quad g=b \prod_{i=1}^{4}\left(z-z_{i}\right)^{3},
$$

where $y, x, \widehat{z}$ are homogeneous coordinates on the fiber and $z$ is the coordinate on the base $\mathbf{P}^{1}$. The K3 described by eq.(2.12) has four $D_{4}$ singularities at $z=z_{i}$. These singularities reproduce the non-abelian gauge symmetry $S O(8)^{4}$ from 4 D7branes on each of the 4 orientifold planes, for generic $U$ and $S$.

Roughly speaking, the complex structure $U$ is related to the cross ratio of the 4 branch points $z_{i}$ of the section $\widehat{z}=0$, while the coupling $S$ may be identified with the modulus of the elliptic curve obtained by scaling $(y, x, z) \rightarrow\left(\lambda^{3} y, \lambda^{2} x, \lambda z\right)$ with $\lambda \rightarrow \infty$. Up to reparametrizations, there are 16 complex parameters in eq. (2.12) that deform the K3 away from the orientifold limit. These correspond to the 16 independent positions of the D7-branes on $\mathbf{P}^{1}$.

In a simple approximation near the orientifold limit, one can think of $\widetilde{X}_{V}$ as an orbifold $X^{b}=T^{2} \times T^{2} / \mathbf{Z}_{2}$. This is not entirely correct, as $X^{b}$ has the wrong vanishing cohomology and therefore cannot describe correctly the D-brane states of the orientifold ${ }^{3}$. As long as we are only interested in the bulk fields, we can still use $X^{b}$ as a model and rewrite the 4 -form flux $G_{4}$ as [1] [2]

$$
G_{4}=G_{3} d \bar{w}+\text { c.c., } \quad G_{3}=G_{z} d z+G_{\bar{z}} d \bar{z} .
$$

Here $d w=d x+S d y$ and $d z=d x^{\prime}+U d y^{\prime}$ are the complex coordinates with $x^{(\prime)}, y^{(\prime)}$ real coordinates with period one on $T^{2} \times T^{2}$. The RR and NS components of the type IIB $G_{3}$ flux are then identified as

$$
G_{3}=F_{3}-S_{3} .
$$

\footnotetext{
3 A different orbifold is discussed in app. B.
} 
The bulk potential (1.1) thus leads to the following expression for the superpotential:

$$
W=\int \Omega \wedge G_{3}=p_{1}+p_{2} U+p_{3} S+p_{4} S U,
$$

with $\Omega=\omega \wedge d z$ and

$$
p_{\Lambda}=\int \omega \wedge \alpha_{\Lambda}, \quad \alpha_{\Lambda}=\eta_{I} c^{I}{ }_{\Lambda}, \quad \Lambda=1, \ldots, 4 .
$$

Eq. (2.14) is the superpotential that has been used in the analysis of [13].

On the other hand, the K3 periods on the orbifold reduce to a simple product of the periods on the two tori

$$
\widetilde{\Pi}^{\Lambda} \sim(1, S) \times(1, U)=(1, S, U, S U) \simeq(S U(1,1) / U(1))^{2} .
$$

Thus the bulk superpotential (2.14) agrees with the dependence of the F-theory potential (2.9) on the fluxes $\underline{\mathrm{G}}^{I \Lambda}, \Lambda=1, \ldots, 4$ and these F-theory fluxes are identified with fluxes of the 3 -form bulk fields $H$ and $F$ in the orientifold limit.

\subsection{Superpotential from 2-form fluxes on D7-branes}

Compared to the potential (2.14), the general superpotential (2.9) depends on 16 additional fluxes. As already noted in [2], these fluxes should also have a natural interpretation in the orientifold, as gauge 2-form fluxes

$$
\mathcal{F}^{a}=\eta^{I} \underline{\mathrm{G}}_{I, a+4}, \quad a=1, \ldots, 16,
$$

on the 16 D7-branes.

Let us substantiate this claim by giving a different derivation of the superpotential on the 7-brane in the presence of 2-form flux, similar to the discussion of D5-branes in [14]. Consider the B-model of the type II string on the 3-fold $Y$, with a B-type brane wrapping $Y$. The gauge theory on the brane is governed by the holomorphic Chern-Simons action [15]:

$$
W=\int_{Y} \Omega \wedge \operatorname{Tr}\left(A \wedge \bar{\partial} A+\frac{2}{3} A \wedge A \wedge A\right) .
$$

The action on the D7-brane, wrapping a 4-dimensional divisor $D \subset Y$, can be computed by dimensional reduction, keeping in mind that the resulting theory on the brane is topologically twisted. The reduction is more complicated as in the D5 case because of the non-trivial dilaton background. However, near the orientifold 
limit $T^{2} / \mathbf{Z}_{2} \times \mathrm{K} 3$, one has approximately a constant dilaton on a Calabi-Yau 3-fold. The complex transverse scalar $v$ is a section of the normal bundle $N(D) \sim \wedge^{2} T^{*}(D)$, where $T^{*}(D)$ denotes the cotangent bundle. In fact $N(K 3)$ is trivial, and $v$ gets identified with one of the deformations $C^{a}$ in (2.8) away from the orientifold limit. The dimensional reduction yields

$$
W_{D 7^{a}}=\int_{D 7^{a}} C^{a} \mathcal{F}^{a} \wedge \omega
$$

where we used $\Omega=\omega \wedge d z$. Thus the holomorphic Chern-Simons action reduced on each of the D7-branes indeed reproduces the 16 extra terms in (2.9).

The general situation with varying dilaton background is more complicated to compute and probably best described directly in F-theory. However, one can extend the above computation to the case of non-trivial normal bundle, with essentially the same result, but with $\omega$ replaced by a residuum of $\Omega$ along $D$. This is very similar as in the computations of D5-brane superpotentials in [14][17], and in fact closely related by the fact that a non-zero superpotential (2.18) for a $(0,2)$ flux $F$ may be attributed to an induced D5-brane wrapping a non-holomorphic 2-cycle in $Y$.

It is important to note however, that the above distinction into bulk and brane fields (and fluxes) is special to the orientifold limit. In general, the 18 complex fields $S, U$ and $C^{a}$ determine locally a point on the coset manifold

$$
\frac{S O(2,18)}{S O(2) \times S O(18)} \supset(S U(1,1) / U(1))_{S} \times(S U(1,1) / U(1))_{U}
$$

which describes the complex structure of the elliptically fibered F-theory K3, $\widetilde{X}_{V}$ [6]. The parametrization (2.8) displays explicitly the embedding of the coset of bulk fields (2.16) at the orientifold point $C^{a}=0$. However, as will be discussed in sect. 3, when we study the vacuum equations, the 2-form fluxes on the D7-branes destabilize the orientifold and drive the branes to generic positions. For general values of the fields, the distinction into bulk and D7-brane moduli is no meaningful notion because of the strong back-reaction of the geometry and the dilaton. A similar comment applies to the distinction into 4 bulk fluxes and 16 brane fluxes.

\subsection{Orbifold limit of $X_{H}$}

On the other hand, one can further simplify the orientifold limit by considering the special case, where also the "lower" $\mathrm{K} 3, X_{H}$, is described by an orbifold. This

\footnotetext{
4 A similar result has been obtained from the BI action in [16].
} 
makes contact to the large literature on intersecting branes on toroidal orbifolds, see the reviews [18] and references therein.

The untwisted moduli space of $Z_{N}$ orbifolds has been well studied and is described by certain cosets described in [19]. K3 orbifolds are included in this classification as the $\mathcal{N}=2$ supersymmetric orbifold sectors with fixed tori. We will discuss the orbifold limit in detail in sects. 5 and 6 , where we compute the effective action including the soft-breaking terms. Here we restrict to display the content of the formula (2.9) for the simple situation, where $X_{H}$ is the orbifold $T^{2} \times T^{2} / \mathbf{Z}_{2}$. As discussed already, the two complex structure moduli $U_{i}$ are described by setting $W^{a}=0$ in (2.8). Plugging $\Pi$ and $\widetilde{\Pi}$ into (2.9) one gets 5

$$
\begin{aligned}
& W_{\left(T^{2} \times T^{2} / \mathbf{Z}_{2}\right)}=1\left(\underline{\mathrm{G}}_{11}+\underline{\mathrm{G}}_{12}\left(\frac{1}{2} C^{a} C^{a}-U S\right)+\underline{\mathrm{G}}_{13} S+\underline{\mathrm{G}}_{14} U+\underline{\mathrm{G}}_{1(a+4)} C^{a}\right)- \\
& U_{1} U_{2}\left(\underline{\mathrm{G}}_{21}+\underline{\mathrm{G}}_{22}\left(\frac{1}{2} C^{a} C^{a}-U S\right)+\underline{\mathrm{G}}_{23} S+\underline{\mathrm{G}}_{24} U+\underline{\mathrm{G}}_{2(a+4)} C^{a}\right)+ \\
& U_{1}\left(\underline{\mathrm{G}}_{31}+\underline{\mathrm{G}}_{32}\left(\frac{1}{2} C^{a} C^{a}-U S\right)+\underline{\mathrm{G}}_{33} S+\underline{\mathrm{G}}_{34} U+\underline{\mathrm{G}}_{3(a+4)} C^{a}\right)+ \\
& U_{2}\left(\underline{\mathrm{G}}_{41}+\underline{\mathrm{G}}_{42}\left(\frac{1}{2} C^{a} C^{a}-U S\right)+\underline{\mathrm{G}}_{43} S+\underline{\mathrm{G}}_{44} U+\underline{\mathrm{G}}_{4(a+4)} C^{a}\right) .
\end{aligned}
$$

At $C^{a}=0$, this is precisely the superpotential that has appeared in the orbifold literature on intersecting branes [20]. Equation (2.20) generalizes their bulk superpotential to include also the moduli $C^{a}$ of the D7-branes. As discussed in (2.17), the fluxes $a>4$ with linear coefficients in the $C^{a}$ describe the a special subset of the 2-form fluxes $\mathcal{F}^{a}$ on the D7-branes, with $I=1, \ldots, 4$. However we will argue below that these fluxes destabilize the orientifold and can not be incorporated consistently within the orientifold/orbifold model.

\section{Minimization of the effective $4 \mathrm{~d}$ potential}

\subsection{F-theory potentials in four dimensions}

Before we turn to a study of the $4 \mathrm{~d}$ vacua with fluxes, it is instructive to

5 We are again oversimplifying here, similar as in the case of the orientifold limit. Although one may describe the $\mathbf{Z}_{2}$ orbifold by a "period vector" as in (2.8), the latter is not defined on a basis that generates $H^{2}\left(X_{H}, \mathbf{Z}\right)$ of the orbifold over the integers. As a consequence, the entries of the matrix $\underline{G}$ appearing in the following equation are not integers but fractional linear combinations of integers. The integrality properties can be reconstructed from the explicit transformation from the integral basis to the orbifold basis, see app. B for more details. 
understand some general properties of the two superpotentials

$$
W=\int_{X_{H}} \omega \wedge \eta^{I} \underline{\mathrm{G}}_{I \Lambda} \int_{\widetilde{X}_{V}} \widetilde{\omega} \wedge \widetilde{\eta}^{\Lambda}, \quad \breve{W}=\int_{X_{H}} j \wedge \eta^{I} \underline{\mathrm{G}}_{I \Lambda} \int_{\widetilde{X}_{V}} \widetilde{j} \wedge \widetilde{\eta}^{\Lambda}
$$

Since the potential $W$ is complex, whereas $\widehat{W}$ is a real function, one would like to identify tentatively $W$ with a 4 d $F$-term and $\widehat{W}$ with a $D$-term. However the definition of the potentials in (3.1) is based on the distinction between Kähler and complex structure moduli of K3, which is an ambigous concept because of the $S U(2)$ symmetry that rotates the three 2 -forms (2.3) into each other.

In the four-dimensional F-theory limit this ambiguity is resolved by the zero elliptic fiber limit of the "upper" K3, $\widetilde{X}_{V}$. The holomorphic elliptic fibration of $\widetilde{X}_{V}$ selects unambigously a complex stucture $\widetilde{\omega}$ and a Kähler structure $\widetilde{j}$. Moreover all Kähler classes of $\widetilde{X}_{V}$ tend to zero in the F-theory limit of zero size fiber, and thus the value of $\breve{W}$ is strictly zero for the fluxes orthogonal to the classes $E$ and $B$ of the fibration in eq.(2.6). The $4 \mathrm{~d}$ scalar potential therefore gets contributions only from the variations $d \breve{W}$ as in (2.11), which is compatible with the interpretation of as a $\Pi^{I, 3} \underline{\mathrm{G}}_{I \Lambda}$ as a $D$-term in the $\Lambda$-th $U(1)$ factor. More precisely, a 2-flux proportional to a 2 -form $\widetilde{\eta}_{\Lambda}$ couples to the difference of two $U(1)$ factors associated with two D7-branes.

The small fiber limit does not resolve a similar ambiguity in the definition of Kähler and complex structure deformations on the "lower" $\mathrm{K} 3 X_{H}$. This reflects the fact that these fields are not decoupled in the potentials and moreover each flux contributes always to both, $F$ - and $D$-terms. It is also clear that one can not distinguish $F$ - and $D$-terms by the Hodge decomposition on $H^{2}\left(X_{H}\right)$.

In fact, in the compactification without flux, the $S U(2)$ that rotates the complex structure on $X_{H}$ is an $R$-symmetry and the only effect that breaks this symmetry is the flux potential itself. We are thus led to define the Hodge structure on $X_{H}$ by the projection to $H^{2}\left(X_{H}\right)$ of the Hodge structure on the 4-form cohomology $H^{4}\left(X_{4}\right) \supset G_{4}$ at the minimum. Since the Hodge type of $G_{4}$ is $(2,2)$ at the minimum [7], the $(2,0)$-form on $X_{H}$ can be defined as the projection $\mu$ of the component

$$
\widehat{G}_{4}=\mu \wedge \overline{\widetilde{\omega}}
$$

onto $H^{2}\left(X_{H}\right)$. With this definition, we can associate the complex potential $W$ with a $F$-term which may give an obstruction to the first order deformations corresponding to elements in

$$
H^{2,0}\left(X_{H}\right) \otimes H^{1,1}\left(\widetilde{X}_{V}\right) \oplus H^{1,1}\left(X_{H}\right) \otimes H^{2,0}\left(\widetilde{X}_{V}\right) \subset H^{3,1}\left(X_{4}\right)
$$


The real $D$-term potential $\widehat{W}$ may obstruct, at first order, only the deformations in

$$
H^{1,1}\left(X_{H}\right) \otimes H^{1,1}\left(\tilde{X}_{V}\right) \subset H^{2,2}\left(X_{4}\right)
$$

Note that deformations of both type of potentials involve 2-forms of type $H^{1,1}\left(X_{H}\right)$, reflecting the fact that the complex structure and Kähler moduli from $X_{H}$ are not decoupled.

Deformations that preserve the complex structure

In a concrete physical application, it is sometimes important to classify the fluxes that preserve a certain complex structure. E.g., the orientifold models correspond to a special complex structure where the D7-branes sit on top of orientifold planes. This is only a consistent restriction if the flux potential does not destabilize the orientifold limit. The same problem appears in orbifold models of K3 that describe only a few of the possible directions of the potential.

The above equations can also be interpreted as saying, that at first order, complex stucture moduli get a potential from a flux component of Hodge type (3.2), while Kähler moduli get a potential from a component of type (3.3). In fact, at second order in the deformation, this distinction is lost and each flux component contributes to both potentials, or $F$ - and $D$-terms. However if we switch on a "new", extra flux component

$$
G_{4}^{*} \in H^{1,1}\left(X_{H}\right) \times H^{1,1}\left(\widetilde{X}_{V}\right)
$$

of type (3.3) in addition to $G_{4}$, the contribution to the $F$-potential will be of the form

$$
\delta W\left(G_{4}^{*}\right) \sim \phi^{2}
$$

where $\phi$ denotes collectively the complex structure moduli with value $\phi=0$ at the original flux vacuum. Thus an extra flux of pure type (3.3) will preserve the complex structure at the minimum of the original potential $W$. The above situation may be visualized in terms of the integral flux matrix $\underline{G}$ as

$$
\underline{\mathrm{G}}_{I \Lambda}=\left(\begin{array}{cc|c}
\widehat{\mathrm{G}}_{3 \times 2}^{++} & \widehat{\mathrm{G}}_{3 \times n^{\prime}}^{+-} & 0 \\
\widehat{\mathrm{G}}_{n \times 2}^{++} & \widehat{\underline{\mathrm{G}}}_{n \times n^{\prime}}^{--} & 0 \\
\hline 0 & 0 & \underline{\mathrm{F}}_{m \times m^{\prime}}^{--}
\end{array}\right),
$$

where we ordered the orthonormal basis $\left\{\eta_{I}\right\}$ for $H^{2}\left(X_{H}\right)$ such that the elements with positive self-intersection are at the first positions and similarly for the basis 
$\left\{\widetilde{\eta}_{\Lambda}\right\}$ for $H^{2}\left(\widetilde{X}_{V}\right)$. The vectors defined by the left upper corner $\underline{\widehat{\mathrm{G}}}$ span a flux space $\mathcal{G}_{\text {flux }} \subset H^{4}\left(X_{4}, \mathbf{R}\right)$ which maps into subspaces of signature $(3, n)$ and $\left(2, n^{\prime}\right)$ under the projection to $H^{2}\left(X_{H}\right)$ and $H^{2}\left(\widetilde{X}_{V}\right)$, respectively. Similarly the right lower corner $\underline{\mathrm{F}}$ can be associated with an orthogonal subspace $\mathcal{F}_{\text {flux }}$ which maps to subspaces of $H^{2}\left(X_{i}\right)$ of purely negative signature under the projection to the K3 cohomology.

In the above notation, an extra flux $G_{4}^{*}$ that preserves the complex structure at the minimum of the flux potential defined by $\underline{\widehat{G}}$, must be of the type $\underline{F}$. Note that the extra flux $G_{4}^{*}$ fulfills the usual quantization conditions and projects to integral $(1,1)$ forms on both K3 factors. A necessary condition for the existence of this type of flux is therefore, that $\operatorname{Pic}\left(X_{H}\right)$ is non-empty and that $\operatorname{dim}\left(\operatorname{Pic}\left(\widetilde{X}_{V}\right)\right)>2$.

Although $G_{4}^{*}$ does not change the minimum for the complex structure moduli, the $D$-term condition $G_{4}^{*} \wedge j=0$ may impose new conditions on the Kähler moduli:

$$
\delta d \breve{W}\left(G_{4}^{*}\right)=\Pi^{I, 3} \underline{\mathrm{F}}_{I \Lambda}=0 .
$$

Let us discuss briefly two important examples of $D$-term conditions. If $\operatorname{Pic}\left(X_{H}\right)$ is empty, there are still non-trivial $D$-term conditions from the flux of type $\widehat{\widehat{G}}$ in (3.5). A universal contribution may arise from a flux aligned with $j$ on $X_{H}$; however, without further contributions to the potential, it drives the theory to the boundary of the moduli space. A more interesting case with a minimum in the interior of the moduli space is the following one. Let $e_{i} \in H^{2}\left(X_{H}\right)$ be the duals of exceptional divisors in the resolution of an orbifold singularity of $K 3$. At the singular point, $\omega \wedge e_{i}=0$ and the Kähler form can grow components in the directions $e_{i}$. There is a moduli space of Kähler structures with Kähler forms

$$
j^{\prime}\left(T^{i}\right)=j+\sum_{i} T^{i} e_{i}, \quad e_{i} \in \operatorname{Pic}\left(X_{H}\right), T^{i} \in \mathbf{R},
$$

and Kähler moduli $T^{i}=\Pi^{i, 3}=\int_{e_{i}^{*}} j^{\prime}$. A non-zero flux with components in the directions of $e_{i}$ induces a $D$-term potential $d \breve{W}$ which imposes again a linear relation

$$
\sum T^{i} \underline{\mathrm{G}}_{i \Lambda}=0
$$

on the volumes of the $e_{i}$. Note that this function is an arbitrary linear combination of the volumes of the exceptional divisors, and the zero does not mean that an individual exceptional divisor shrinks at the minimum; therefore the generic minimum is not at an geometric orbifold point in the moduli. 


\subsection{General structure of minima}

We discuss now in more detail the vacuum conditions imposed by the potential arising from $W$. The equations

$$
D_{n} W=\left(\partial_{n}+\partial_{n} K\right) W=0
$$

describe minima at a vanishing value of the potential. Here $n$ runs over all complex hyper and vector moduli6 in the periods $\Pi$ and $\widetilde{\Pi}$, respectively, and $K$ is the Kähler potential. The number of equations is equal to the number of moduli and thus all moduli appearing in $W$ are fixed in a generic solution.

It is straightforward to compute the content of (3.8) for the potential (2.5). The result is that if the solution is in the interior of the moduli space, the 4-form flux $G_{4}$ decomposes into pieces that are sums of forms of definite Hodge type

$$
G_{4}=G_{0}+G_{1}+G_{2}, \quad\left\{\begin{array}{l}
G_{0} \in H_{B}^{2,0} \wedge H_{F}^{2,0}+\text { c.c. } \\
G_{1} \in H_{B}^{2,0} \wedge H_{F}^{0,2}+\text { c.c. } \\
G_{2} \in H_{B}^{1,1} \wedge H_{F}^{1,1}
\end{array}\right.
$$

whereas the components in (3.2) are set to zero by the constraints (3.8). The potential thus reproduces part of the general vacuum conditions of [7] and was designed to do so in [1].

The general configuration describes a non-supersymmetric vacuum with vanishing potential. There are also two supersymmetric branches of the vacuum space, characterized by the vanishing of components in the decomposition (3.9). As there are no more moduli to tune, a vanishing of these components can only be achieved by treating the entries of the flux matrix $\underline{G}_{I \Lambda}$ as further parameters; however such a tuning may be in conflict with the flux quantization. Neglecting this problem for the moment one finds the following three different branches:

$$
\begin{array}{ll}
\mathcal{N}=0: & \text { generic }, \\
\mathcal{N}=1: & G_{0}=0, \\
\mathcal{N}=2: & G_{0}=G_{1}=0 .
\end{array}
$$

Each constraint $G_{a}=0, a=0,1$ gives two real conditions on the real matrix $\underline{\mathrm{G}}_{I \Lambda}$. The $\mathcal{N}=1$ supersymmetric branch is characterized by the vanishing of $G_{0}$, where $W=0$ in addition to $(3.8)$.

6 From F-theory perspective it is natural to include the dilaton amongst the complex structure moduli and we do so in the following. 
On the other hand, for $G_{1}=G_{0}=0$, the flux $G_{4}$ is of type $(1,1)$ on both K3's. If it is primitive as well, it is orthogonal to all 2-forms $\omega^{i}$ in eq.(2.3). Thus it is a primitive $(2,2)$ flux w.r.t. to the $\mathbf{P}^{1} \times \mathbf{P}^{1}$ of complex structures of the Mtheory compactification without flux, which is a sufficient criterion for extended supersymmetry [2]. In the decomposition of the flux matrix (3.5), this corresponds to the case $\underline{\widehat{G}}=0, \underline{F} \neq 0$.

\subsection{Vacuum solutions}

To determine the $\mathcal{N}=1$ supersymmetric vacua, we consider the potential (2.9) in the form,

$$
W=\int_{X_{H}} \omega \wedge g_{2,0}, \quad g_{2,0}=\eta_{I} \underline{\mathrm{G}}_{\Lambda}^{I} \widetilde{\Pi}^{\Lambda} .
$$

Since $D_{i} \omega$ is a form of pure type $(1,1)$, the equations $W=D_{i} W=0$ imply that $g_{2,0}$ is of type $(2,0)$. Comparing this with the expansion $\omega=\Pi^{I} \eta_{I}$, we find

$$
\Pi^{I}=c \underline{\mathrm{G}}_{\Lambda}^{I} \widetilde{\Pi}^{\Lambda}, \quad c \in \mathbf{C}
$$

at the vacuum. Inserting this into the quadratic constraint $\omega \wedge \omega=0$, one finds the following equation, which is equivalent to $W=0$ :

$$
\widetilde{\Pi}^{\Sigma} \underline{\mathrm{G}}^{I} \underline{\mathrm{G}}_{I \Lambda} \widetilde{\Pi}^{\Lambda}=0=\widetilde{\Pi}^{\Lambda} \widetilde{M}_{\Sigma \Lambda} \widetilde{\Pi}^{\Lambda} .
$$

On the r.h.s. we have written the quadratic constraint on the periods of $\widetilde{X}_{V}$ to illustrate the two basic ways to solve this equation. A special solution is

$$
\underline{\mathrm{G}}_{\Lambda \Sigma}=\widetilde{M}_{\Lambda \Sigma} \longleftrightarrow \Pi^{I} \sim \widetilde{\Pi}^{\Lambda}
$$

This is a symmetric solution, where the two K3 factors are at the same modulif. Note that this solution to (3.11) has a moduli space corresponding to the complex structure of one elliptic K3.

The above branch of solutions arises from the quadratic constraint satisfied by the periods for all values of the moduli. The other possibility to satisfy (3.11) is if $\widetilde{\Pi}^{\Lambda}$ is a null vector of $\underline{G}$ at special values of the moduli. The generic solution is a combination of both solutions, that is the contribution of non-zero vectors must be proportional to the quadratic constraint.

7 In particular, $X_{H}$ has an elliptic fibration and the non-zero period vector has dimension 20, as for $\widetilde{X}_{V}$. 
To determine all moduli, one may use the Hodge decomposition (3.9), explicitly:

$$
\begin{aligned}
g_{2,0} & =\eta_{I} \underline{\mathrm{G}}^{I}{ }_{\Lambda} \widetilde{\Pi}^{\Lambda}, \\
g_{1,1}^{a} & =\eta_{I} \underline{\mathrm{G}}^{I}{ }_{\Lambda} D_{a} \widetilde{\Pi}^{\Lambda}, \\
g_{0,2} & =\overline{g_{2,0}},
\end{aligned}
$$

where $D_{a}=\partial_{a}+K_{a}$ are the covariant derivatives w.r.t. the complex moduli of $\widetilde{X}_{V}$. The values of the moduli at the vacuum are then determined by eq.(3.11) and the equations

$$
g_{2,0} \wedge g_{1,1}^{a}=g_{2,0} \wedge \overline{g_{1,1}^{a}}=0 .
$$

For the supersymmetric vacua satisfying (3.11), these equations simplify considerably as one may replace the covariant derivatives by ordinary ones and moreover the ordinary derivatives are linear in the period vector

$$
\partial_{a} \widetilde{\Pi}^{I}=\left(X_{a}\right)^{I}{ }_{J} \widetilde{\Pi}^{J}
$$

for a constant matrix $X_{a}$. The equations (3.11),(3.14) represents a system of holomorphic polynomials of degree $\leq 4$ that is straightforward to solve. We will discuss some examples below.

$\mathcal{N}=2$ vacua

The $\mathcal{N}=2$ vacua correspond to $G_{1}=G_{0}=0$, or

$$
g_{2,0}=\eta_{I} \underline{G}_{\Lambda}^{I} \widetilde{\Pi}^{\Lambda}=0,
$$

which is equivalent to $\omega \wedge G_{4}=\widetilde{\omega} \wedge G_{4}=0$. This is possible only if $\underline{G}$ is a sum of products of anti-selfdual forms on the two K3 factors. The space of $\mathcal{N}=2$ vacua has various branches labeled by $r=\operatorname{rank} \underline{\mathrm{G}} \leq 18$. The residual geometric moduli space on the branch labelled by the integer $r$ is generically

$$
\mathbf{R}_{+} \times \frac{S O(3,19-r)}{S O(3) \times S O(19-r)} \times \frac{S O(2,18-r)}{S O(2) \times S O(18-r)} \times \mathbf{R}_{+} .
$$

Note that the fact that $\omega \perp G_{4}$ does not necessarily mean that the $\mathcal{N}=2$ solutions are orbifolds, since the classes appearing in $G_{4}$ may have non-zero Kähler volumes. In fact the generic $D$-term potential (2.11) has a minimum that fixes the Kähler volumes away from the orbifold. 
Non-supersymmetric vacua

Non-supersymmetric vacua are described by solutions to eq. (3.14) that do not solve eq.(3.11). These vacuum equations are no longer holomorphic polynomials, but can still be solved for specific examples.

\subsection{Examples}

We will now study some features of solutions to the general potential (2.9) in some examples. As discussed already, the equations $W=D_{i} W=0$, where $i$ is a modulus of $X_{H}$, are solved by $g_{2,0} \in H^{2,0}\left(X_{H}\right)$. Differentiating the first line of (2.9) w.r.t. the moduli of $\widetilde{X}_{V}$ one finds, in terms of $p_{\Lambda}=\Pi^{I} \underline{\mathrm{G}}_{I \Lambda}$,

$$
\begin{aligned}
& p_{3}-p_{2} U=0, \quad p_{4+a}+p_{2} C_{a}=0 \text {, } \\
& p_{4}-p_{2} S=0, \quad p_{1}+p_{2}\left(U S-\frac{1}{2} C^{a} C^{a}\right)=0 \text {. }
\end{aligned}
$$

The two branches of solutions to the above equations are

$$
\begin{array}{lll}
\underline{\mathcal{N}=2:} & p_{\Lambda}=0 & g_{2,0}=0 . \\
\underline{\mathcal{N}=1:} & p_{2} \neq 0 & U=\frac{p_{3}}{p_{2}}, \quad S=\frac{p_{4}}{p_{2}},
\end{array}
$$

The above formula displays already two important properties of the generic solution on the $\mathcal{N}=1$ supersymmetric branch:

i) for generic flux, all $D 7$ moduli $C^{a}$ are fixed.

ii) For generic flux, the $D 7$ branes are stabilized at generic positions $C^{a} \neq 0$ away from the orientifold. Thus the orientifold is not a 'preferred' point in the space of vacua and the generic flux destabilizes the orientifold geometry.

8 Near the orientifold limit, this follows from the local analysis presented in [10]. 


\section{Orientifold/orbifold compactification}

As the first concrete example consider the orientifold. Shifting coordinates as in (B.2) and after a basis transformation, one obtains the "period vector" $\widetilde{\Pi}^{\Lambda}$ in eq.(B.3) for the orientifold, with the orientifold point described in the shifted variables as $C^{a}=0$. Similarly one may use a transformed "period vector" $\Pi^{I}$ for the "lower" $\mathrm{K} 3, X_{H}$, such that the point $W^{i}=0$ corresponds to a point in the moduli of the $\mathrm{K} 3$ orbifold $T^{4} / \mathbf{Z}_{2}$. Although the following discussion does not rely on assuming an orbifold limit for $X_{H}$, it it is instructive to do so to compare with the explicit discussion in sect. 5 .

In the orientifold/orbifold basis for the period vectors, switch on a flux $\underline{G}$ corresponding to a plane $\mathcal{G}_{\text {flux }}$ with signature $(2,2)$ defined by $\underline{\mathrm{G}}_{I \Lambda}=0$ for $I>$ $5, \Lambda>4$. The potential is given by (2.9), with all $p_{\Lambda}, \Lambda>4$ zero. The minimization of the potential is then as in [13], except for two differences. First recall that the entries of $\underline{G}$ in this basis are not integers, but fractional linear combinations of integers that can be determined by the transformation to the integral basis (A.7). Secondly the potential has an additional quadratic piece in the $D 7$ brane moduli

$$
\delta W=\frac{1}{2} p_{2} \sum_{a}\left(C^{a}\right)^{2}
$$

which sets $C^{a}=0$ for all $a$ if $p_{2} \neq 0$. Recall that $p_{2}=0$ corresponds to the $\mathcal{N}=2$ supersymmetric branch, so the $D 7$-branes are fixed to the orientifold plane by this choice of bulk flux on the $\mathcal{N}=1$ supersymmetric branch. Similarly, the minimum for the moduli of $X_{H}$ is at the orientifold point $W^{i}=0$.

As discussed before, a generic flux will destabilize the orientifold. However, as discussed in sect. 3.1, one can also add another flux to this compactification, without destabilizing the orientifold. Consider an additional flux $\underline{F}$ corresponding to another plane $\mathcal{F}_{\text {flux }}$ such that $\mathcal{G}_{\text {flux }}$ and $\mathcal{F}_{\text {flux }}$ are orthogonal, that is $\underline{\mathrm{F}}_{I \Lambda}=0$ for $I \leq 5, \Lambda \leq 4.9$ This flux corresponds to a 2 -form flux

$$
\mathcal{F}^{a}=\underline{\mathrm{F}}_{i a} \eta^{i}
$$

on the $D 7$ branes. In the above basis, there is an extra superpotential of the type (3.4),

$$
W^{\prime}=\sum W^{i} C^{a} \underline{\mathrm{F}}_{i a},
$$

whose value and derivatives are zero at the minimum of the original potential $W$. Thus a 2 -form flux $\mathcal{F}^{a}$ of the type $\underline{\mathrm{F}}$ induces a superpotential that does not destabilize the orientifold/orbifold vacuum.

9 As explained earlier, this requires that $\operatorname{Pic}\left(X_{H}\right)$ is non-empty. 
In addition to the superpotential $W^{\prime}$, the same flux $\mathcal{F}^{a}$ induces an extra $D$-term potential (3.6). By definition, this $D$-term restricts Kähler moduli on $X_{H}$

$$
d \breve{W}^{\prime}=T^{i} \underline{\mathrm{F}}_{i a}=0, \quad T^{i}=\Pi^{3, i} \in \mathbf{R} .
$$

Some more examples with 2-form flux

In the following we illustrate some of the features of the general potential at the hand of some simple examples. The fluxes in these examples are given in the orthonormal basis for both $K 3$ factors, corresponding to the period vectors in the form (A.7).

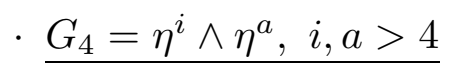

This choice for $G_{4}$ corresponds to a pure 2-form flux $\mathcal{F}^{a}=\eta^{i}$ on the $a$-th brane with $\operatorname{rank}(\underline{\mathrm{G}})=1$ (and is the special case of the above example, with $\underline{\mathrm{G}} \rightarrow$ $0, \underline{\mathrm{F}} \rightarrow \underline{\mathrm{G}})$. The superpotential is $W=W^{i} \cdot C^{a}$, with a $\mathcal{N}=2$ supersymmetric minimum at $W^{i}=C^{a}=0$. The Hodge type of $\mathcal{F}^{a}$ at the minimum is $(1,1)$. The same flux induces both, the $F$-term potential $W$ as well as a $D$-term potential with minimum $T^{i}=0$. This is the simplest example to illustrate that all components of the 2 -form flux on the brane enter both potentials.

- $G_{4}=\eta^{i} \wedge \eta^{4}, i>4$

This is again a $\operatorname{rank}(\underline{\mathrm{G}})=1$ example, which however restricts the bulk fields. The superpotential is $W=W^{i} \cdot(S-U)$ with an $\mathcal{N}=2$ supersymmetric minimum at $W^{i}=0=S-U$. There is also a $D$-term that requires $j \perp \eta^{i}$. Note that this is a supersymmetric flux vacuum with bulk fluxes where nevertheless all the D7-brane moduli are massless. This illustrates that supersymmetric bulk fluxes of type $(2,1)$ need not give masses for the $D 7$ moduli, differently then in previous examples presented in [10] [21].

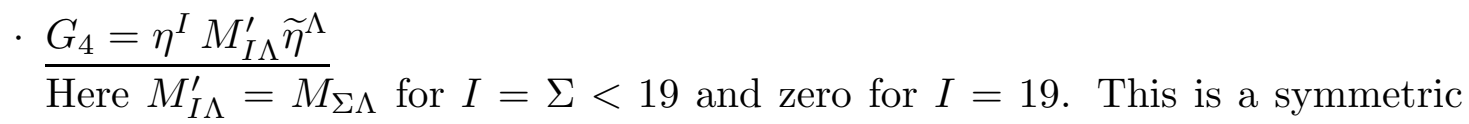
solution, where both K3 factors are elliptically fibered and at the same complex structure moduli. The vacuum has $\mathcal{N}=1$ supersymmetry and a large complex structure moduli space, corresponding to one elliptically fibered K3. 
Change of hodge type of the supersymmetric flux

In the case of only a bulk potential (11.1) with a supersymmetric minimum, the Hodge type of the 3 -form flux $G_{3}$ is $(2,1)$. This is also reflected in the computations of mass terms on the brane [21,22].

In the generic configuration with general fluxes and D7-branes at arbitrary positions, there is no obvious notion of what one calls a flux of Hodge type $(2,1)$. However one can ask the simpler question, whether at least under a small deformation of the $D 7$ branes away from the orientifold, the supersymmetric bulk flux remains of type $(2,1)$. In fact there is no reason to expect this and indeed the following short computation shows that the Hodge type changes already at first order in the deformation. This should also be relevant for computations of soft terms on the world volume of D-branes away from the orientifold plane.

The Hodge decomposition for the bulk flux is, in the notation of sect. 2.5,

$$
G_{4}=G_{3} d \bar{w}+G_{3}^{*} d w=\left(G_{z} d z+G_{\bar{z}} d \bar{z}\right) d \bar{w}+\left(G_{z}^{*} d \bar{z}+G_{\bar{z}}^{*} d z\right) d w
$$

At the supersymmetric minimum, $G_{3}$ is of type $(2,1)$ and thus $G_{z}$ is of type $(1,1)$ and $G_{\bar{z}}$ of type $(2,0)$. To include the 2 -form fluxes, we first write the Hodge decomposition in terms of the $K 3$ periods as

$$
\int G_{4} \wedge \widetilde{\omega}=G_{\bar{z}} \Pi+G_{z} \Pi_{U}+\text { c.c. } .
$$

where $\Pi_{a}=D_{a} \Pi$ projects to a form of pure type $(1,1)$ on the "lower" K3 $X_{H}$. To determine the effect of a small perturbation it suffices to consider the case with a single 2-form flux. The Hodge decomposition of the 4-form flux becomes

$$
\int G_{4} \wedge \widetilde{\omega}=G_{\bar{z}}^{\prime} \Pi^{\prime}+G_{z}^{\prime} \Pi_{U}^{\prime}+\mathcal{F}^{a} \Pi_{C^{a}}^{\prime}+\text { c.c. },
$$

where the prime indicates the periods at $v \neq 0$ and $\mathcal{F}^{a}$ is identified with a non-zero 2 -form flux on the brane that induces the deformation. In the vacuum, $G_{\bar{z}}^{\prime}$ is of type $(2,0)$ while $G_{z}^{\prime}$ and $\mathcal{F}^{a}$ are of type $(1,1)$.

To compute the change of Hodge type linearly in the deformation $C^{a}$, one may rewrite $G_{4}$ in terms of the original Hodge decomposition; at the required order, it is consistent to keep the complex structure of $T^{2}$ constant. The coefficient of the 2 -form $d \bar{z} d \bar{w}$ is

$$
G_{\bar{z}}^{\prime}+\frac{1}{4 S_{2} U_{2}} \bar{C}^{a}\left(\mathcal{F}^{a}+\overline{\mathcal{F}}^{a}\right)
$$

Thus the supersymmetric bulk flux is no longer of pure type $(2,1)$ but has a piece of Hodge type $(1,2)$ at first order in the deformation. 


\section{Embedding in supergravity}

Below we rephrase the flux potential (2.9) in the language of the effective $4 \mathrm{~d}$ supergravity and give a direct relation between the flux parameters in (2.2) and certain gaugings in supergravity. Aspects of the effective supergravity for the orientifold limit have been discussed in [2] [13] and in particular in [23] [24].

\subsection{Effective supergravity}

Let us first recall briefly the $\mathcal{N}=2$ effective supergravity for the bulk fields of the orientifold $T^{2} / \mathbf{Z}_{2} \times \mathrm{K} 3$ from ref.[23]. There are 4 vector fields from reducing the ten-dimensional 2-forms $B_{N S}$ and $B_{R R}$ with one leg along $T^{2}$. Three of them become part of $4 \mathrm{~d}$ vector multiplets with scalar components

$$
\begin{aligned}
S & =C_{0}+i e^{-\phi_{10}}, \\
T & =\int_{K 3} C_{4}+i e^{-\phi_{10}} \operatorname{Vol}(K 3), \\
U & =\frac{1}{G_{11}}\left(G_{12}+i \sqrt{\operatorname{det} G}\right),
\end{aligned}
$$

where $G_{m n}$ is the metric on $T^{2} / \mathbf{Z}_{2}, C_{p}$ denotes the RR $p$-form and $S$ is the complex type IIB dilaton 10 The bulk scalars parametrize locally the special Kähler manifold

$$
\mathcal{M}_{V}^{0}=(S U(1,1) / U(1))_{S, U, T}^{3} .
$$

The hyper multiplet moduli space

$$
\mathcal{M}_{H}=O\left(\Gamma^{4,20}\right) \backslash S O(4,20) / S O(4) \times S O(20),
$$

is associated with the moduli of $K 3$ up to one subtlety. In the decomposition

$$
S O(4,20) \simeq S O(3,19) \times \mathbf{R}^{22} \times \mathbf{R}_{+},
$$

the coset associated with the first factor describes the Einstein metrics of constant volume discussed before and the second factor the $B$-fields on $H^{2}(K 3)$. However, the last factor describes the Kähler volume of $T^{2}$ [23].

One may readily identify a $(S U(1,1) / U(1))_{S, U}^{2}$ factor parametrized by the $8 \mathrm{~d}$ fields $S$ and $U$ in $\mathcal{M}_{V}^{0}$ as the subset (2.16) of the F-theory compactification. However

$10 \operatorname{Vol}(K 3)$ denotes the volume of K3 in the string frame. 
the full $8 \mathrm{~d}$ F-theory spectrum, including the brane gauge fields and the moduli scalars $C^{a}$, is given by the complex structure moduli in (2.19). The enlarged special Kähler manifold that includes the three bulk fields (4.2) as well as the brane degrees of freedom is therefore 11

$$
\mathcal{M}_{V}=\frac{S U(1,1)}{U(1)} \times \frac{S O(2,18)}{S O(2) \times S O(18)} .
$$

A similar proposal, including also possible moduli from D3 branes, has been made in [24] based on constraints from electric-magnetic duality. However as discussed around eq.(4.10), the F-theory derivation adds some important extra physical information related to the integral lattice of BPS states and the D7-brane positions.

\subsection{Supergravity gaugings vs. flux potentials}

We will now give the precise dictionary between the F-theory fluxes in the superpotential (2.9) and the gaugings in the effective supergravity. We will use the notations of [26], to which we also refer for background material on gauged supergravities.

In the formalism of $\mathcal{N}=2$ supergravity, the scalar potential, for purely electric gauging, is given by the expression

$$
V=4 h_{u v} k_{\Lambda}^{u} k_{\Sigma}^{v} L^{\Lambda} \bar{L}^{\Sigma}+\left(U^{\Lambda \Sigma}-3 \bar{L}^{\Lambda} L^{\Sigma}\right) P_{\Lambda}^{x} P_{\Sigma}^{x}
$$

where

$$
U^{\Lambda \Sigma}=-\frac{1}{2}\left(\operatorname{Im} \mathcal{N}_{\Lambda \Sigma}\right)^{-1}-\bar{L}^{\Lambda} L^{\Sigma}, \quad L^{\Lambda}=e^{K_{V} / 2} X^{\Lambda}
$$

Here $\left(X^{\Lambda}, F_{\Lambda}\right), \Lambda=0, \ldots, n_{V}$ denotes the symplectic section that defines the special geometry of the $n_{V}$ vector multiplets, $\mathcal{N}_{\Lambda \Sigma}$ is the coupling matrix of the gauge fields and $K_{V}$ is the Kähler potential on $\mathcal{M}_{V}$. Moreover $h_{u v}$ is the metric for the hyper multiplets. The couplings between hyper multiplets and vector multiplets are described by the Killing vectors $k_{\Lambda}^{u}$ and the associated prepotentials $P_{\Lambda}^{x}$. The index $x$ labels a triplet under the $S U(2)$ symmetry, which is embedded in the holonomy of the quaternionic manifold $\mathcal{M}_{H}$.

Under certain conditions, the above scalar potential can be related to the scalar potential of an $\mathcal{N}=1$ supergravity obtained from $F$-terms and $D$-terms. To do so,

11 Another quick way to get the following moduli space is to use the 8-dimensional duality [6] between F-theory on $\mathrm{K} 3$ and the heterotic string on $T^{2}$, and the moduli space for the perturbative heterotic string compactified on $T^{2} \times \mathrm{K} 3[25]$. 
one can try to split the contributions to $V$ into a candidate $F$-term related to, say, the complex prepotentials

$$
P_{\Lambda}=P_{\Lambda}^{1}+i P_{\Lambda}^{2},
$$

and a $D$-term related to $P_{\Lambda}^{3}$, or another choice related to it by $S U(2)$ rotations. The candidate expression for the holomorphic superpotential of $\mathcal{N}=1$ supergravity for the flux potential (1.1) is then [5] 27]

$$
W=\sum_{\Lambda} P_{\Lambda}^{\prime} X^{\Lambda}-\sum_{\Lambda} \widetilde{P}^{\prime \Lambda} F_{\Lambda}
$$

where the tilded prepotentials $\widetilde{P^{\Lambda}}$ describe the coupling of the dual magnetic fields and the prime denotes the holomorphic sections, related to $P_{\Lambda}$ by the standard exponential in the Kähler potential. The ansatz (4.7) is justified, if the scalar potential $V$ can be written as a standard $\mathcal{N}=1$ potential based on $W$ and an additional $D$-term that depends on the real prepotential $P_{\Lambda}^{3}$.

Dependence on $\mathcal{M}_{H}$

The manifold $\mathcal{M}_{H}$ has translational isometries corresponding to shifts of the $22 \mathrm{~B}$ fields on K3, made explicit in the decomposition (4.4). For these "axionic" gaugings there is a simple relation between the prepotentials and the Killing vectors

$$
P_{\Lambda}^{x}=\omega_{I}^{x} k_{\Lambda}^{I},
$$

where $\omega_{I}^{x}$ is the $S U(2)$ connection [28]. It will turn out, that the objects $\omega_{I}^{x}$ are essentially the periods $\Pi_{I}^{x}$ defined in (2.4), up to an overall factor. One has

$$
\omega_{I}^{x} \sim e^{\alpha}\left(L^{-1}\right)_{I}^{x},
$$

where $L$ is a coset representative of $S O(3,19) / S O(3) \times S O(19)$ and $\alpha$ parametrizes the volume factor [23].

For the purpose of defining the superpotential (4.7), we must choose now one direction in $S U(2)$, say $x=3$, and define the complex holomorphic prepotential (4.6). In the present case this is the same as choosing a fixed complex structure on the "lower" $X_{H}$ and this is already anticipated by the split (2.1) of the Mtheory superpotential. As discussed in sect. 3.1, the superpotential $W$ becomes the 4-dimensional $F$-term while $d \breve{W}$ will enters a $D$-term in the $\mathcal{N}=1$ sense.

Accordingly, we introduce the complex quantities

$$
\omega_{I} \equiv \omega_{I}^{0}+i \omega_{I}^{1} \sim e^{\alpha} L^{J} M_{J I}, \quad L^{I}=L_{1}^{I}+i L_{2}^{I},
$$


where we used $L^{T} M L=M$.

Finally, it follows from $L^{I} M_{I J} \bar{L}^{J}=1$ that $L^{I}=e^{K_{0} / 2} \Pi^{I}$, with $\Pi^{I}$ the holomorphic period vector and $K_{0}$ the Kähler potential for the coset based on $S O(3,19)$. The final form for the complex Killing prepotential (4.6) is then

$$
P_{\Lambda}=e^{K_{H} / 2} \Pi^{I} M_{I J} k_{\Lambda}^{J},
$$

where $K_{H}=K_{0}+2 \alpha$ is the Kähler potential on $\mathcal{M}_{H}$ and a similar formula holds for the real prepotential $P_{\Lambda}^{3}$.

Dependence on $\mathcal{M}_{V}$

The defining data of the effective supergravity for the $n_{V}$ is the symplectic section $\left(X^{\Lambda}, F_{\Lambda}\right)$. As explained in [24], the geometry of the 8-dimensional vector multiplets is unaffected by the compactification on the "lower" $\mathrm{K} 3, X_{H}$. This implies that the symplectic section is given by a product of the 8 -dimensional period vector $\widetilde{\Pi}_{\Lambda}$ in (2.8) and the period vector $(1, T)$ for the volume of $X_{H}: 12$

$$
X^{\Lambda}=\left(\begin{array}{c}
1 \\
-S U+\frac{1}{2} C^{a} C^{a} \\
S \\
U \\
C^{a}
\end{array}\right), \quad F_{\Lambda}=T M_{\Lambda \Sigma} \widetilde{\Pi}^{\Sigma} .
$$

Note that the F-theory derivation of the section $X^{\Lambda}=\widetilde{\Pi}^{\Lambda}$, as the period vector of the "upper" K3, provides important physical information that does not follow from the duality argument in supergravity used in [24]. Specifically the period vector is defined with reference to the integral lattice $H^{2}\left(\widetilde{X}_{V}, \mathbf{Z}\right)$, which represents the lattice of BPS charges arising from the 20 8-dimensional $U(1)$ gauge fields. In this context, $X^{\Lambda}$ represents the central charge of $4 \mathrm{~d}$ BPS domain walls carrying a certain gauge charge.

Without reference to the integral lattice, the supergravity section $X^{\Lambda}$ cannot determine the D7-brane positions. In fact any point on $\mathcal{M}_{V}$ can be parametrized locally by a section (4.10) with $C^{a}=0$ and $S, U$ some complex numbers. E.g., the different physics of D7-branes at generic positions on one side, and D7-branes on top of the orientifold plane on the other side, is described by the different sets of integral vectors orthogonal to the section $X^{\Lambda}$ at, say, $C^{a}=0$. Similarly the integrality properties of the flux, or the gaugings in supergravity, refer to a symplectic section in the integral basis, which is provided by the geometry of the F-theory compactification.

12 To make contact with the standard supergravity conventions, one must transform the period vector $\widetilde{\Pi}^{\Lambda}$ into the orthonormal basis (A.7). 
Mapping fluxes and gaugings

Combining the above equations, the candidate superpotential becomes a degree $(1,1)$ polynomial in the periods of the two K3's:

$$
W=\sum_{\Lambda} P_{\Lambda}^{\prime} X^{\Lambda}=\sum_{I \Lambda} \Pi^{I} \widetilde{\Pi}^{\Sigma} k_{I \Lambda}
$$

where we removed the exponential $e^{K_{H} / 2}$ to pass to the holomorphic section $P_{\Lambda}^{\prime}$ and $k_{I \Lambda}=M_{I J} k_{\Lambda}^{I}$. Note that $W$ is also an inhomogeneous polynomial of degree 4 in the K3 moduli.

The expression (4.11) is in perfect agreement with (2.5) if we identify the Ftheory fluxes (2.2) and the Killing vectors $k_{\Lambda}^{I}$ as

$$
\underline{\mathrm{G}}_{\Lambda}^{I}=k_{\Lambda}^{I}
$$

As already explained in detail in sect. 2.5., near the orientifold, the fluxes with index $\Lambda=1, \ldots 4$ are identified with the bulk 3 -form flux $G_{3}$, while the fluxes with $\Lambda=$ $5, \ldots, 16$ describe the 2 -form flux on the $\mathrm{D} 7$-brane. At the risk of being repetitive, let us summarize this relation between fluxes and Killing vectors near the orientifold limit 13

$$
\begin{array}{rlrl}
k_{1}^{I}=U_{2}^{-1} \int F_{3} \wedge\left(\eta^{I} d x\right) & \text { RR bulk 3-form } \\
k_{4}^{I}=U_{2}^{-1} \int F_{3} \wedge\left(\eta^{I} d y\right) & " \\
k_{2}^{I}=U_{2}^{-1} \int H_{3} \wedge\left(\eta^{I} d y\right) & \text { NS bulk 3-form } \\
k_{3}^{I}=-U_{2}^{-1} \int H_{3} \wedge\left(\eta^{I} d x\right) & & " \\
k_{a}^{I}= & \int F_{2}^{a} \wedge \eta^{I} & & 16 \text { D7-brane 2-forms }(a>4)
\end{array}
$$

The supergravity prepotentials $P_{\Lambda}^{x}$, associated with these fluxes are obtained by contracting these vectors with the $\mathrm{K} 3$ periods as in (4.8).

13 The following identifications refer to the basis (2.8) of the period vector, defined in terms of the integral basis $\left\{\eta^{I}\right\}$ for $H^{2}\left(X_{H}, \mathbf{Z}\right)$; moreover $d z=d x+U d y$ is the complex coordinate on $T^{2} / \mathbf{Z}_{2}$, as before. 


\subsection{Vacua in gauged supergravity}

It is remarkable that the analysis of the vacuum configurations is much simpler in the flux language then in the supergravity language. As an illustration of this point, we use the identification (4.12) to display "new" vacua of the effective gauged supergravity.

In fact it has been known for a while that it is quite hard to find $\mathcal{N}=1$ supersymmetric vacua by gauging a $\mathcal{N}=2$ supergravity. There is a no-go theorem 229 that says that this is impossible if the section $\left(X^{\Lambda}, F_{\Lambda}\right)$ can be derived from a prepotential $\mathcal{F}$ such that $F_{\Lambda}=\partial \mathcal{F} / \partial X^{\Lambda}$. The non-existence of a prepotential is a very strong condition which requires a special scalar manifold $\mathcal{M}_{V}$ and in addition a special choice for the symplectic section $\left(X^{\Lambda}, F_{\Lambda}\right)$ [30]. Remarkably enough, the above map from the flux potential to a gauged supergravity has precisely led to a special section (4.10) that can not be derived from a prepotential. This is consistent with the existence of $\mathcal{N}=1$ supersymmetric vacua, reported in [13] and sect. 3 .

As a simple example let us first reconsider the bulk moduli of the orientifold. The 4-dimensional slice (2.16) of the orientifold has already been study from the two different perspectives, namely using the superpotential (1.1) in [13] and using gauged supergravity in [23], however without an explicit comparison. The flux superpotential obtained from (1.1) in 113 is

$$
W \sim \int \omega \wedge\left(-\alpha_{y}+S \beta_{y}+U \alpha_{x}-S U \beta_{x}\right) \equiv \int \omega \wedge \mu
$$

where $\alpha_{a}, \beta_{a} \in H^{2}(K 3, \mathbf{Z})$ are defined by the 3 -form fluxes

$$
F_{3}=\alpha_{x} d x+\alpha_{y} d y, \quad H_{3}=\beta_{x} d x+\beta_{y} d y .
$$

On the other hand, the supergravity potential is obtained from (4.7) with the period vector (2.8) transformed to the orthogonal basis with metric $\operatorname{diag}(1,1,-1,-1)$

$$
X^{\Lambda}=\frac{1}{\sqrt{2}}\left(\begin{array}{c}
1-S U \\
-S-U \\
-(1+S U) \\
U-S
\end{array}\right)=\left.\frac{1}{\sqrt{2}}\left(\begin{array}{cccc}
1 & 1 & 0 & 0 \\
0 & 0 & -1 & -1 \\
1 & -1 & 0 & 0 \\
0 & 0 & -1 & 1
\end{array}\right) \widetilde{\Pi}\right|_{C^{a}=0}
$$

The explicit mapping (4.12) from fluxes to Killing vectors $k_{\Lambda}^{I}$ of the gauged supergravity is 14 :

$$
\begin{array}{ll}
\alpha_{x}=\frac{1}{\sqrt{2}}\left(-k_{1}^{I}+k_{3}^{I}\right) \eta_{I}, & \alpha_{y}=\frac{1}{\sqrt{2}}\left(-k_{0}^{I}+k_{2}^{I}\right) \eta_{I}, \\
\beta_{x}=\frac{1}{\sqrt{2}}\left(k_{0}^{I}+k_{2}^{I}\right) \eta_{I}, & \beta_{y}=\frac{1}{\sqrt{2}}\left(-k_{1}^{I}-k_{3}^{I}\right) \eta_{I},
\end{array}
$$

14 In this formula we use the supergravity notation of [23] with $\Lambda=0, \ldots, 3$. 
The condition $G_{1}=0$ on the $\mathcal{N}=2$ supersymmetric branch corresponds to the vanishing of the 2 -form $\mu$ in (4.14); this gives two real condition on the four component 2 -forms $\alpha_{a}, \beta_{a}$, or two conditions on the four Killing vectors $k_{\Lambda}^{I}$. E.g., a choice of fluxes that fixes $S$ and $U$ at purely imaginary values is

$$
k_{0}^{I}=\frac{1-S_{2} U_{2}}{1+S_{2} U_{2}} k_{2}^{I}, \quad k_{1}^{I}=\frac{U_{2}-S_{2}}{U_{2}+S_{2}} k_{3}^{I},
$$

where $S=S_{1}+i S_{2}$ and similarly for $U$. More generally, scanning through complex vev's for $S$ and $U$ one obtains a family parametrized by four real parameters that contains the special $\mathcal{N}=2$ supersymmetric solution found in [23].

Gauged supergravity vacua with 2-form fluxes

In [24], duality arguments have been used to propose an effective supergravity description for the type IIB string on $T^{2} / \mathbf{Z}_{2} \times \mathrm{K} 3$, including D7- and D3-brane moduli. In the absence of D3-branes and setting $n_{V}=18$, this manifold reduces to the $4 \mathrm{~d}$ F-theory moduli space (4.5).

As discussed already, F-theory provides extra physical information, such as the integral structure defined by the lattice of BPS states. Moreover it gives a string theory interpretation of the extra 16 Killing prepotentials in (4.13), as 2-form fluxes on the 16 D7-branes. The vacuum structure for special choice of Killing vectors has also been studied in [24], with the result that the D7-branes are always confined to the origin $C^{a}=0$. This is somewhat at odds with the claim of sect. 3 , that the generic flux fixes the D7-branes at generic position $C^{a} \neq 0$.

However, using the flux-gauging dictionary in (4.13), it is straightforward to check that the choice of Killing vectors in [24] corresponds to the special situation, where the plane associated with the 2 -form flux $\mathcal{F}_{\text {flux }}$ is orthogonal to the plane $\mathcal{G}_{\text {flux }}$ defined by the bulk flux. Thus the vacua of [24] fit into the classification of the orientifold preserving fluxes in sect. 3.3, in agreement with the fact that $C^{a}=0$ at the minimum.

\section{Superpotential and open string moduli in orbifold models}

In this and the next section we study in depth the type $I I B$ orientifold $K 3 \times$ $T^{2} / \Omega$ in the case where the $\mathrm{K} 3$ is the orbifold $T^{4} / \mathbf{Z}_{2}$. In particular we describe the full superpotential including open string moduli and 2-form fluxes and derive the effective gauged supergravity describing the orbifold. 


\subsection{The type IIB $K 3 \times T^{2} / \Omega$ orientifold}

\section{Geometrical moduli}

First, consider the case type IIB on $K 3 \times T^{2}$ without the orientifold projection. The corresponding moduli space takes the form:

$$
\mathcal{M}=\frac{S O(22,6)}{S O(22) \times S O(6)} \otimes\left(\frac{S U(1,1)}{U(1)}\right)_{U} .
$$

The first factor parametrizes the $\sigma$-model manifold of 132 scalar fields, which are the scalar components of $22 \mathrm{~N}=4$ vector supermultiplets. Among them there are the 57 Kähler and complex structure moduli at constant volume of $K 3$, the type $I I B$ dilaton, the two volume moduli of $K 3$ and $T^{2}$ plus several other Ramond-Ramond scalar fields. Note that the modulus $U$, which parametrizes the second factor in $E q$. (5.1) and which corresponds to the complex structure of $T^{2}$, is a member of the $\mathrm{N}=4$ supergravity multiplet.

Now, we introduce the orientifold projection $\Omega(-1)^{F_{L}} I_{2}^{3}$, with $I_{2}^{3}$ acting on the complex torus coordinate $z^{3}$ as: $I_{2}^{3}: z^{3} \rightarrow-z^{3}$. This gives an $\mathrm{N}=2$ closed string spectrum. The geometrical $\mathrm{N}=4$ moduli space in $E q$. (5.1) gets truncated and factorizes locally into a space of $\mathrm{N}=2$ vector- times $\mathrm{N}=2$ hypermultiplets [23]:

$$
\mathcal{M}=\mathcal{M}_{V}^{0} \otimes \mathcal{M}_{H},
$$

with $\mathcal{M}_{V}^{0}$ the special Kähler manifold for the moduli (4.1) given in (4.2). The metric of these fields is described by the Kähler potential

$$
K_{V}=-\ln (S-\bar{S})(T-\bar{T})(U-\bar{U}),
$$

which is derived from the following $\mathrm{N}=2$ prepotential:

$$
\mathcal{F}(S, T, U)=S T U \text {. }
$$

The geometric moduli space of $K 3$ is described by the coset space

$$
\frac{S O(19,3)}{S O(19) \times S O(3) \times S O(19,3, \mathbf{Z})} \times \mathbf{R}^{+}
$$

with real dimensions 58 , while the stringy moduli space of $K 3$ is given by the coset in (4.3), except for the exchange of volume factors from $T^{2}$ and $K 3$ [23]. The additional 22 scalars represent the 22 axions $\left(b^{m}, b^{a}\right), m=1,2,3, a=1, \ldots, 19$. They originate from reducing the $R R 4$-form on $K 3 \times T^{2}$ : Due to $h_{(2,0)}(K 3)=$ 
$h_{(0,2)}(K 3)=1$ and $h_{(1,1)}(K 3)=20$, we obtain the two fields $C_{m n 3 \overline{3}}$ and $C_{\overline{m n} 3 \overline{3}}$ and the 20 fields $C_{m \bar{n} 3 \overline{3}}$ with the index 3 referring to the $(1,1)$-form of the torus $T^{2,3}$. Note, that in $D=4$ these scalars are dual to the anti-symmetric tensors $C_{m n \mu \nu}, C_{\overline{m n} \mu \nu}$ and the 20 fields $C_{m \bar{n} \mu \nu}$, respectively. The latter are eliminated as a result of imposing self-duality on the self-dual 4 -form $C_{4}$. At any rate, we thus obtain 22 scalars from the 4 -form $C_{4}$ reduced w.r.t. the cohomology $H^{2}(K 3)$. The $80 K 3$-moduli appear as scalars of $20 \mathrm{~N}=2$ hypermultiplets. Furthermore, due to $h_{(0,0)}(K 3)=h_{(2,2)}(K 3)=1$ we obtain the field $C_{\mu \nu \rho \sigma}$, which gives rise to a tadpole in $D=4$, and $a^{3}=\int_{K 3} C_{m \bar{n} p \bar{q}}$ representing the axion of the Kähler modulus of the torus $T^{2,3}$. The latter is dual to the anti-symmetric tensor $\int_{T^{2}} C_{\mu \nu 3 \overline{3}}$, which is eliminated after imposing self-duality on $C_{4}$.

\section{Adding D3- and D\%-branes}

To cancel the tadpoles we add a system of $n_{3}$ space-time filling $D 3$-branes and $n_{7} D 7$-branes wrapped on $K 3$. On the internal $D 7$-brane world volume non-trivial two-form gauge fluxes $\mathcal{F}^{a}$ may be turned on. The open string $D 7$-brane moduli consist of the transverse positions $C^{7, a}:=C_{3}^{7, a}$ of the $n_{7} D 7$-branes along the torus $T^{2}\left(a=1, \ldots, n_{7}\right)$ and $3 n_{3} D 3$-brane moduli $C_{i}^{3, b}$ accounting for the transverse $D$-brane positions along $K 3 \times T^{2}\left(b=1, \ldots, n_{3}\right)$. The scalars $C_{i}^{3, b}, i=1,2$ give rise to additional hypermultiplet scalars. On the other hand, in addition to the three fields $S, T, U$ we have the $n_{7}+n_{3}$ scalars $C^{7, a}, C^{3, b}:=C_{3}^{3, b}$ of $\mathrm{N}=2$ vectormultiplets in $D=4$. In the following, we shall disregard the open string moduli $C_{i}^{3, b}, i=1,2$ giving rise to additional hypermultiplet scalars.

The metric of these vectormultiplet fields is described by the Kähler potential [31,24, 32]:

$$
\begin{aligned}
K_{V} & =-\ln \left[(S-\bar{S})(T-\bar{T})(U-\bar{U})-\frac{1}{2}(T-\bar{T}) \sum_{a=1}^{n_{7}}\left(C^{7, a}-\bar{C}^{7, a}\right)^{2}\right. \\
& \left.-\frac{1}{2}(S-\bar{S}) \sum_{b=1}^{n_{3}}\left(C^{3, b}-\bar{C}^{3, b}\right)^{2}\right]
\end{aligned}
$$

which is derived from the following $\mathrm{N}=2$ prepotential [24]:

$$
\mathcal{F}\left(S, T, U, C^{7, a}, C^{3, b}\right)=S T U-\frac{1}{2} T \sum_{a=1}^{n_{7}}\left(C^{7, a}\right)^{2}-\frac{1}{2} S \sum_{b=1}^{n_{3}}\left(C^{3, b}\right)^{2} .
$$

On the internal $D 7$-brane world volume the non-trivial (magnetic) two-form gauge flux

$$
\mathcal{F}_{N P}^{a}=\mathcal{F}_{12}^{a}+\mathcal{F}_{34}^{a}:=2 \pi \alpha^{\prime}\left(F_{12}^{a} d x^{1} \wedge d y^{1}+F_{34}^{a} d x^{2} \wedge d y^{2}\right)
$$


may be turned on. The latter obey the quantization rule $F_{i j}^{a}=2 \pi \frac{n_{i j}^{a}}{m_{i j}^{a}}$, i.e. :

$$
f_{i j}^{a}=\frac{1}{(2 \pi)^{2}} \int_{C_{i j}} \mathcal{F}_{i j}^{a}=\alpha^{\prime} \frac{n_{i j}^{a}}{m_{i j}^{a}} \quad, \quad(i, j)=(1,2),(3,4) .
$$

The dependence of the moduli and matter field metrics on these 2-form fluxes has been derived in 20,33:

$$
\begin{aligned}
G_{T \bar{T}} & =-\frac{1}{(T-\bar{T})^{2}} \quad, \quad G_{S \bar{S}}=-\frac{1}{(S-\bar{S})^{2}} \quad, \quad G_{U \bar{U}}=-\frac{1}{(U-\bar{U})^{2}}, \\
G_{C^{7, a} \bar{C}^{7, a}} & =-\frac{1}{(S-\bar{S})(U-\bar{U})}+\frac{\alpha^{\prime-2} f_{12}^{a} f_{34}^{a}}{(T-\bar{T})(U-\bar{U})}, \\
G_{C^{3, b} \bar{C}^{3, b}} & =\frac{-1}{(T-\bar{T})(U-\bar{U})} .
\end{aligned}
$$

In fact, up to second order in the matter fields $C^{7, a}, C^{3, b}$, we may summarize these results in the Kähler potential

$$
\begin{aligned}
K_{V} & =-\ln [(S-\bar{S})(T-\bar{T})(U-\bar{U}) \\
& -\frac{1}{2} \sum_{a=1}^{n_{7}}\left[(T-\bar{T})-(S-\bar{S}) f_{12}^{a} f_{34}^{a} \alpha^{\prime-2}\right]\left(C^{7, a}-\bar{C}^{7, a}\right)^{2} \\
& \left.-\frac{1}{2} \sum_{b=1}^{n_{3}}(S-\bar{S})\left(C^{3, b}-\bar{C}^{3, b}\right)^{2}\right]
\end{aligned}
$$

which is derived from the following $\mathrm{N}=2$ prepotential:

$\mathcal{F}\left(S, T, U, C^{7, a}, C^{3, b}\right)=S T U-\frac{1}{2} \sum_{a=1}^{n_{7}}\left(T-\alpha^{\prime-2} S f_{12}^{a} f_{34}^{a}\right)\left(C^{7, a}\right)^{2}-\frac{1}{2} S \sum_{b=1}^{n_{3}}\left(C^{3, b}\right)^{2}$.

It may be interesting to note, that a similar instanton-number dependent prepotential arises in heterotic $K 3 \times T^{2}$ compactifications 34.

In order to preserve supersymmetry on the $D 7$-brane-world volume, the $2-$ form fluxes have to obey 35

$$
\frac{f_{12}^{a}}{\operatorname{Im} T^{2}}=-\frac{f_{34}^{a}}{\operatorname{Im} T^{1}}
$$

Note that these equations are nothing but the $D$-term equation (3.19). The two fluxes $f_{12}^{a}, f_{34}^{a}$ are quantized according to (5.8). This fixes the ratio $\operatorname{Im} T^{1} / \operatorname{Im} T^{2}$ to

$$
\frac{\operatorname{Im} T^{1}}{\operatorname{Im} T^{2}}=-\frac{n_{34}^{a} m_{12}^{a}}{n_{12}^{a} m_{34}^{a}}
$$

Later we shall see, that these two-form fluxes correspond to a specific gauging in the underlying low-energy supergravity description, which fixes the ratio $\operatorname{Im} T^{1} / \operatorname{Im} T_{2}$. 


\subsection{The moduli space in the orbifold limit $T^{4} / \mathbf{Z}_{2}$ of $K 3$}

In the following, let us work in an orbifold limit of $K 3$. In the case of a $\mathbf{Z}_{2^{-}}$ orbifold limit the hypermultiplet moduli space $\mathcal{M}_{H}$ in (4.3) boils down to the coset

$$
\mathcal{M}_{H}=\frac{S O(4,4)}{S O(4) \times S O(4) \times S O(4,4, \mathbf{Z})},
$$

whose real dimension is 16 . The coset may be described by the symmetric $G$ and anti-symmetric background $B$, with $G^{t}=G$ and $B^{t}=-B$. More precisely, in Narain lattice compactifications one introduces the moduli scalar matrix 1 国

$\mathcal{M}=\frac{1}{2}\left(\begin{array}{ll}G+G^{-1}+G^{-1} B-B G^{-1}-B G^{-1} B & G-G^{-1}+G^{-1} B+B G^{-1}-B G^{-1} B \\ G-G^{-1}-G^{-1} B-B G^{-1}-B G^{-1} B & G+G^{-1}-G^{-1} B+B G^{-1}-B G^{-1} B\end{array}\right)$

describing a general $\frac{S O(d, d)}{S O(d) \times S O(d)}$-background. On may verify $\mathcal{M}^{t}=\mathcal{M}, \operatorname{det}(\mathcal{M})=$ 1 and $\mathcal{M} \eta \mathcal{M}^{t}=\eta$, with $\eta=\operatorname{diag}\left(+1^{d},-1^{d}\right)$. The moduli metric is given by $d s^{2}=-\frac{1}{4} \operatorname{Tr}\left(d \mathcal{M} d \mathcal{M}^{-1}\right)$.

For our case, i.e. $d=4$, the background $G$ and $B$ gives rise to 16 real parameters, which comprise the two complex structure moduli $U^{1}, U^{2}$ and six Kähler moduli $\mathcal{T}^{i}, i=1, \ldots, 6$ of $T^{4} / \mathbf{Z}_{2}$. Note that the split into Kähler and complex structure moduli is only a convention on $K 3$. The Kähler moduli $\mathcal{T}^{i}$ are complexified with the $R R$-axions $a^{i}$ encoded in the anti-symmetric background:

$$
B=\left(\begin{array}{cccc}
0 & a^{1} & a^{3} & a^{4} \\
-a^{1} & 0 & a^{5} & a^{6} \\
-a^{3} & -a^{5} & 0 & a^{2} \\
-a^{4} & -a^{6} & -a^{2} & 0
\end{array}\right)
$$

For the case under consideration (5.14) the decomposition (4.4) translates into:

$$
\frac{S O(4,4)}{S O(4) \times S O(4)}=\frac{S O(3,3)}{S O(3) \times S O(3)} \times \mathbf{R}_{+} \times \mathbf{R}^{6}
$$

15 Note, that alternatively, by the unimodular transformation $\frac{1}{\sqrt{2}}\left(\begin{array}{cc}\mathbf{1}_{d} & \mathbf{1}_{d} \\ -\mathbf{1}_{d} & \mathbf{1}_{d}\end{array}\right)$ one may transform the background $\mathcal{M}$ into the form:

$$
\mathcal{M}=\left(\begin{array}{cc}
G^{-1} & G^{-1} B \\
-B G^{-1} & G-B G^{-1} G
\end{array}\right)
$$


The coset $\frac{S O(3,3)}{S O(3) \times S O(3)}$ describes nine of the ten parameter of $G$, while the factor $\mathbf{R}_{+}$describes the volume of $T^{4}$. In the next section we shall discuss the gauging of the remaining six $\mathrm{PQ}$-symmetries of $\left(b^{m}, b^{a}\right), m, a=1,2,3$, with the following identifications:

$$
\left(\begin{array}{c}
b^{m} \\
b^{a}
\end{array}\right)=\frac{1}{\sqrt{2}}\left(\begin{array}{c}
-a^{4}-a^{5} \\
a^{3}-a^{6} \\
a^{1}+a^{2} \\
a^{4}-a^{5} \\
a^{3}+a^{6} \\
-a^{1}+a^{2}
\end{array}\right) \quad m, a=1,2,3
$$

Generically, the metric of the space (5.14) is given by:

$$
d s_{S O(4,4)}^{2}=-\frac{1}{2} \operatorname{Tr}\left(d G^{-1} d G\right)-\frac{1}{2} \operatorname{Tr}\left(G^{-1} d B G^{-1} d B\right) .
$$

If we specialize the torus $T^{4}$ to be a direct product of two two-tori $T^{4} \simeq T^{2,1} \times T^{2,2}$, with the field-theoretical moduli 16

$$
T^{j}=a^{j}+i \sqrt{\operatorname{det} G^{j}} \quad, \quad U^{j}=\frac{1}{G_{11}^{j}}\left(G_{12}^{j}+i \sqrt{\operatorname{det} G^{j}}\right) \quad, \quad j=1,2,
$$

respectively, the background $G$ and $B$ in (5.19) may be parametrized by

$$
B:=\left(\begin{array}{cc}
B_{1} & B_{3} \\
-B_{3}^{t} & B_{2}
\end{array}\right) \quad, \quad G:=\left(\begin{array}{cc}
G_{1} & 0 \\
0 & G_{2}
\end{array}\right)
$$

with the $2 \times 2$ matrices $B_{i}, i=1,2,3$ and $G_{1}, G_{2}$ :

$$
G_{i}=\frac{T_{2}^{i}}{U_{2}^{i}}\left(\begin{array}{cc}
1 & U_{1}^{i} \\
U_{1}^{i} & \left|U^{i}\right|^{2}
\end{array}\right) \quad, \quad i=1,2
$$

In Eq. (5.22) we have kept all six RR-scalars $a^{j}$ in order to discuss the gauging of all six isometries of (5.18). With the moduli $T^{j}, U^{j}, j=1,2$, the metric may be also written:

$$
d s_{S O(4,4)}^{2}=4 \sum_{M, N \in\left\{T^{i}, U^{j}\right\}} \frac{\partial^{2} K_{H}}{\partial M \partial \bar{N}}+\operatorname{Tr}\left(G_{1}^{-1} d B_{3} G_{2}^{-1} d B_{3}\right),
$$

16 We have the following relations between physical and geometric quantities [33,20]:

$$
\operatorname{Im} T^{i}=\frac{1}{2 \pi \alpha^{\prime 2}} e^{-\phi_{10}} \mathcal{T}_{2}^{j} \mathcal{T}_{2}^{k}=\frac{1}{2 \pi \alpha^{\prime / 2}} e^{-\phi_{4}} \sqrt{\frac{\mathcal{T}_{2}^{j} \mathcal{T}_{2}^{k}}{\mathcal{T}_{2}^{i}}}
$$

Furthermore, the $K 3$ - or $T^{4}$-volume is given by $\operatorname{Vol}(K 3)=\left(2 \pi \alpha^{\prime 2}\right)^{-1} \mathcal{T}_{2}^{1} \mathcal{T}_{2}^{2}$, and the torus volume $\operatorname{Vol}\left(T^{2}\right)=\mathcal{T}_{2}^{3}$. In addition, we have: $\phi_{10}=\phi_{4}+\frac{1}{2} \ln \left(\mathcal{T}_{2}^{1} \mathcal{T}_{2}^{2} \mathcal{T}_{2}^{3} / \alpha^{\prime 3}\right)$. 
with the Kähler potential:

$$
K_{H}=-\sum_{j=1}^{2} \ln \left(U^{j}-\bar{U}^{j}\right)-\sum_{j=1}^{2} \ln \left(T^{j}-\bar{T}^{j}\right) .
$$

In the following we shall introduce new fields such, that in Eq. (5.19) and (5.24) the decomposition (5.17) becomes visible. This is achieved through two steps. We first split off the volume factor $\mathbf{R}_{+}$in (5.17) by introducing the new fields

$$
\begin{aligned}
\rho & =\frac{\operatorname{Im}\left(T^{1}\right)}{\operatorname{Im}\left(T^{2}\right)}=\frac{\operatorname{Im} \mathcal{T}^{2}}{\operatorname{Im} \mathcal{T}^{1}}, \\
e^{\Phi} & =\sqrt{\operatorname{Im}\left(T^{1}\right) \operatorname{Im}\left(T^{2}\right)}=\frac{e^{-\phi_{10}}}{2 \pi \alpha^{\prime 2}} \sqrt{\mathcal{T}_{2}^{1} \mathcal{T}_{2}^{2}} \mathcal{T}_{2}^{3}=\frac{e^{-\phi_{4}}}{2 \pi \alpha^{\prime 1 / 2}} \sqrt{\mathcal{T}_{2}^{3}},
\end{aligned}
$$

with $e^{2 \Phi}$ essentially describing the volume $\operatorname{Vol}\left(T^{2}\right)$ of the torus $T^{2}$, i.e. the factor $\mathbf{R}_{+}$in (5.17). With these new coordinates the metric (5.19) of the coset (5.14) may be written:

$$
\begin{aligned}
d s_{S O(4,4)}^{2} & =-4 \frac{d U^{1} d \bar{U}^{1}}{\left(U^{1}-\bar{U}^{1}\right)^{2}}-4 \frac{d U^{2} d \bar{U}^{2}}{\left(U^{2}-\bar{U}^{2}\right)^{2}}+\frac{1}{2} \frac{d \rho^{2}}{\rho^{2}}+2 d \Phi^{2} \\
& +e^{-2 \Phi}\left[\frac{\left(d a^{1}\right)^{2}}{\rho}+\rho\left(d a^{2}\right)^{2}\right]+\operatorname{Tr}\left(G_{1}^{-1} d B_{3} G_{2}^{-1} d B_{3}\right)
\end{aligned}
$$

As a second step we have to introduce the parametrization of the coset $\frac{S O(3,3)}{S O(3) \times S O(3)}$, which accounts for the remaining metric moduli $U^{1}, U^{2}, \rho$. This has to be achieved such, that the metric of the $R R$-scalars $b^{m}, b^{a}$ takes the appropriate form, dictated by the decomposition (5.17).

In the supergravity literature, see Ref. [26] and in particular the Refs. [23, 24, the local parametrization of the moduli space $\frac{S O(4,4)}{S O(4) \times S O(4)}$ according to the decomposition (5.17) is given by the coset representative $e^{C^{I} Z_{I}} e^{\phi S} L$, with $Z_{I}$ the generators of the Abelian shift symmetries on $b^{I}, S$ a generator of $S O(1,1)$ and $L$ being a coset representative of $S O(3,3)$.

Generically, the coset representative $L$ of the manifold $\frac{S O(d, d)}{S O(d) \times S O(d)}$ is parametrized by the $d^{2}$ coordinates $e=\left\{e_{m}{ }^{a}\right\}, e^{t}=\left\{e_{a}{ }^{m}\right\}, m, a=1, \ldots, d$

$$
L_{M}^{A}=\left(\begin{array}{cc}
\sqrt{1+e e^{t}} & e \\
e^{t} & \sqrt{1+e^{t} e}
\end{array}\right),
$$

with $L \eta L^{t}=\eta$, implying $L_{M}{ }^{A} \eta_{A B} L_{N}{ }^{B}=\eta_{M N}$. Any $S O(d, d)$ background $\mathcal{M}$, described by $G$ and $B$ as given in $E q$. (5.15), may be expressed by

$$
\mathcal{M}=L^{t} L
$$


with the matrix representation:

$$
L=\frac{1}{2}\left(\begin{array}{ll}
q+q^{*}+q^{*} B & q-q^{*}+q^{*} B \\
q-q^{*}-q^{*} B & q+q^{*}-q^{*} B
\end{array}\right) .
$$

Here, $q$ is the vielbein $q_{m a}$, with $G_{m n}=\delta^{a b} q_{m a} q_{n b}$, i.e. $G=q q^{t}$ and $q^{*}=\left(q^{t}\right)^{-1}$. To relate the matrix representation (5.30) to the coordinates (5.28), we find for the parametrization of $S O(3,3)$ :

$$
e_{m}^{a} \equiv e=\frac{1}{2}\left(q-q^{*}+q^{*} B\right)
$$

On may verify, that $\operatorname{det}(L)=1$ and $L \eta L^{t}=\eta$. The last matrix identity implies $\frac{1}{4}\left(q+q^{*}+q^{*} B\right)\left(q+q^{*}+q^{*} B\right)^{t}-e e^{t}=1$, which translates into: $\sqrt{1+e e^{t}}{ }_{m}^{n} \equiv$ $\sqrt{1+e e^{t}}=\frac{1}{2}\left(q+q^{*}+q^{*} B\right)$.

After this general discussion about the parametrization of coset space in supergravity, let us go back to our metric (5.27) to be decomposed according to (5.17). The parametrization $L$ of the coset $\frac{S O(3,3)}{S O(3) \times S O(3)}$, accounting for the remaining metric moduli $U^{1}, U^{2}, \rho$ of $T^{4}$ may be found as follows. Since we restrict the torus $T^{4}$ to a direct product of two two-tori, the coset $S O(3,3)_{\rho, U^{1}, U^{2}}$ decomposes into the product $S O(2,2)_{U^{1}, U^{2}} \times S O(1,1)_{\rho}$. The $S O(2,2)$-structure of $e$ is obtained from (5.31), with the choice:

$$
G=\frac{U_{2}^{2}}{U_{2}^{1}}\left(\begin{array}{cc}
1 & U_{1}^{1} \\
U_{1}^{1} & \left|U^{1}\right|^{2}
\end{array}\right) \quad, \quad B=\left(\begin{array}{cc}
0 & U_{1}^{2} \\
-U_{1}^{2} & 0
\end{array}\right)
$$

i.e.:

$$
q_{S O(2,2)}=\sqrt{\frac{U_{2}^{2}}{U_{2}^{1}}}\left(\begin{array}{ll}
1 & U_{1}^{1} \\
0 & U_{2}^{1}
\end{array}\right)
$$

Furthermore, with

$$
q_{S O(1,1)}=\sqrt{\rho}
$$

we find:

$$
e=\left(\begin{array}{ccc}
\frac{-U^{1}+\bar{U}^{1}+U^{2}-\bar{U}^{2}}{2 \sqrt{U^{1}-\bar{U}^{1}} \sqrt{U^{2}-\bar{U}^{2}}} & \frac{U^{1} U^{2}-\bar{U}^{1} \bar{U}^{2}}{2 \sqrt{U^{1}-\bar{U}^{1}} \sqrt{U^{2}-\bar{U}^{2}}} & 0 \\
\frac{i\left(U^{1}+\bar{U}^{1}-U^{2}-\bar{U}^{2}\right)}{2 \sqrt{U^{1}-\bar{U}^{1}} \sqrt{U^{2}-\bar{U}^{2}}} & -\frac{i\left(2+U^{1} U^{2}+\bar{U}^{1} \bar{U}^{2}\right)}{2 \sqrt{U^{1}-\bar{U}^{1}} \sqrt{U^{2}-\bar{U}^{2}}} & 0 \\
0 & 0 & \frac{1}{2}\left(\sqrt{\rho}-\frac{1}{\sqrt{\rho}}\right)
\end{array}\right) .
$$

Since $S O(2,2)_{U^{1}, U^{2}} \simeq S U(1,1)_{U^{1}} \times S U(1,1)_{U^{2}}$, we could have also transformed $e$ into a complex basis, where the moduli $U^{1}$ and $U^{2}$ are decoupled. The latter basis is in fact the period vector $\Pi^{I}$ at the orientifold point and the $3 \times 3$ matrix $e$ above is 
essentially, up to an overall factor, the triplet of period vectors $\Pi_{I}^{x}$ (2.4), restricted to $S O(2,2) \times S O(1,1) \subset S O(3,19)$.

To this end, we find the $S O(4,4)$ metric (5.27) in terms of $L(e), \Phi$ and the Ramond fields $b^{m}, b^{a}$ :

$$
d s_{S O(4,4)}^{2}=-\frac{1}{4} \operatorname{Tr}\left[d\left(L^{t} L\right) d\left(L^{-1} L^{*}\right)\right]+2 d \Phi^{2}+e^{-2 \Phi}\left(\begin{array}{c}
d b^{m} \\
d b^{a}
\end{array}\right)^{t} L^{t} L\left(\begin{array}{c}
d b^{m} \\
d b^{a}
\end{array}\right) .
$$

Note, that the modulus $\Phi$, introduced in $E q$. (5.26), plays the rôle of the extra factor $\mathbf{R}_{+} \equiv S O(1,1)$ in the decomposition (5.17).

\subsection{Superpotential from gauged supergravity}

Turning on fluxes in string theory may be understood from the supergravity point of view as gauging some of the isometries of the moduli space of the scalars under some of the gauge fields $A_{\mu}^{\Lambda}$ of the vectormultiplets $\Lambda=0,1,2, \ldots, n_{3}+n_{7}+3$. More precisely, the relevant isometries in the case under consideration are associated to Peccei-Quinn-symmetries of the scalars $\left(b^{m}, b^{a}\right)$, which originate from the $C_{4}$ $R R$-form:

$$
\begin{aligned}
D_{\mu} b^{m} & =\partial_{\mu} b^{m}+f_{\Lambda}^{m} A_{\mu}^{\Lambda}, \\
D_{\mu} b^{a} & =\partial_{\mu} b^{a}+h_{\Lambda}^{a} A_{\mu}^{\Lambda} .
\end{aligned}
$$

In type IIB string theory, a subset of these couplings $f_{\Lambda}^{m}, h_{\Lambda}^{a}$ correspond to nonvanishing $N S$ and $R$ three-form fluxes and non-trivial two-form fluxes on the $D 7$-brane world-volume. In the above we have used the following notations of Ref. [24] for the Killing vectors $k_{\Lambda}^{I}$,

$$
k_{\Lambda}^{I}=\left\{\begin{array}{ll}
f_{\Lambda}^{m} & I=m=1,2,3 \\
h_{\Lambda}^{a} & I-2=a=1,2,3
\end{array},\right.
$$

which distinguish between directions of different signature. The conformal Killing potentials $P_{\Lambda}^{x}, x=1,2,3$, are expressed in Ref. [24] as

$$
P_{\Lambda}=\sqrt{2} e^{-\Phi}\left[\left(L^{-1}\right)_{m}^{x} f_{\Lambda}^{m}+\left(L^{-1}\right)_{a}^{x} h_{\Lambda}^{a}\right]=\sqrt{2} e^{-\Phi} L^{-1}\left(\begin{array}{c}
f_{\Lambda}^{m} \\
h_{\Lambda}^{a}
\end{array}\right)
$$


With our parametrization (5.34) we obtain:

$$
\begin{aligned}
& P_{\Lambda}^{1}=\frac{e^{-\Phi}}{\sqrt{2\left(U^{1}-\bar{U}^{1}\right)\left(U^{2}-\bar{U}^{2}\right)}}\left[f_{\Lambda}^{1}\left(U^{1}-\bar{U}^{1}+U^{2}-\bar{U}^{2}\right)+h_{\Lambda}^{1}\left(U^{1}-\bar{U}^{1}-U^{2}+\bar{U}^{2}\right)\right. \\
& \left.+\left(f_{\Lambda}^{2}-h_{\Lambda}^{2}\right)\left(U^{1} U^{2}-\bar{U}^{1} \bar{U}^{2}\right)\right], \\
& P_{\Lambda}^{2}=-\frac{i e^{-\Phi}}{\sqrt{2\left(U^{1}-\bar{U}^{1}\right)\left(U^{2}-\bar{U}^{2}\right)}}\left[f_{\Lambda}^{1}\left(U^{1}+\bar{U}^{1}+U^{2}+\bar{U}^{2}\right)+h_{\Lambda}^{1}\left(U^{1}+\bar{U}^{1}-U^{2}-\bar{U}^{2}\right)\right. \\
& \left.+f_{\Lambda}^{2}\left(U^{1} U^{2}+\bar{U}^{1} \bar{U}^{2}-2\right)-h_{\Lambda}^{2}\left(U^{1} U^{2}+\bar{U}^{1} \bar{U}^{2}+2\right)\right], \\
& P_{\Lambda}^{3}=\frac{1}{\sqrt{2}} e^{-\Phi}\left[\left(f_{\Lambda}^{3}+h_{\Lambda}^{3}\right) \frac{1}{\sqrt{\rho}}+\left(f_{\Lambda}^{3}-h_{\Lambda}^{3}\right) \sqrt{\rho}\right] \text {. }
\end{aligned}
$$

The (electrical part of) $\mathrm{N}=1$ superpotential $W, E q$. (4.7), is:

$$
W=2^{-3 / 2} e^{-K_{H} / 2} X^{\Lambda}\left(P^{1}+i P^{2}\right)_{\Lambda} .
$$

Here, the parameter $f_{\Lambda}^{m}, h_{\Lambda}^{a}$ refer to the electrical gaugings, introduced in $E q$. (5.36). Furthermore, the symplectic section $\left(X^{\Lambda}, F_{\Lambda}\right)$ in the orthonormal basis, with $X^{\Lambda}$ given by

$$
\begin{aligned}
& X^{0}=\frac{1}{\sqrt{2}}\left[1-S U+\frac{1}{2} \sum_{a=1}^{n_{7}}\left(C^{7, a}\right)^{2}\right], \\
& X^{1}=-\frac{1}{\sqrt{2}}(S+U), \\
& X^{2}=-\frac{1}{\sqrt{2}}\left[1+S U-\frac{1}{2} \sum_{a=1}^{n_{7}}\left(C^{7, a}\right)^{2}\right], \\
& X^{3}=-\frac{1}{\sqrt{2}}(U-S), \\
& X^{a}=C^{7, a} \\
& X^{b}=C^{3, b}
\end{aligned}
$$

is obtained from (5.11) and the special coordinates after a symplectic transformation [24]. This transformation leads to sections with the property $F_{\Lambda} \sim T$, which is appropriate for our D7-brane setup. Note, that that symplectic transformation, given in [24], may be also used for the case of non-vanishing $f_{12}^{a}, f_{34}^{a}$ two-form flux components. As a result, the flux dependence will only enter the sections $F_{\Lambda}$. 
With the relation $e^{-K_{H} / 2}=2 i e^{\Phi} \sqrt{\left(U^{1}-\bar{U}^{1}\right)\left(U^{2}-\bar{U}^{2}\right)}$, we find for $(5.39)$ :

$$
\begin{aligned}
W\left(S, T, U^{1}, U^{2}, U^{3}, C^{7}, C^{3}\right) & =\sum_{\Lambda=0}^{4+n_{7}+n_{3}} X^{\Lambda}\left\{f_{\Lambda}^{1}\left(U^{1}+U^{2}\right)-f_{\Lambda}^{2}\left(1-U^{1} U^{2}\right)\right. \\
& \left.+h_{\Lambda}^{1}\left(U^{1}-U^{2}\right)-h_{\Lambda}^{2}\left(1+U^{1} U^{2}\right)\right\}
\end{aligned}
$$

with the sections $X^{\Lambda}$ given in $(5.40)$ and the identification $U \equiv U^{3}$. This agrees with the F-theory superpotential $(2.20)$ for $C^{3, b}=0$. Note, that, in addition, there appear the couplings to the sections $F_{\Lambda}$ as a matter of symplectic invariance. The latter are proportional to the $K 3$-volume modulus $T$, i.e. $F_{\Lambda} \sim T$.

\subsection{Discussion of Superpotential: two-form and three-form fluxes}

In this subsection we shall further explore the relation (4.12) between the different directions of gaugings $f_{\Lambda}^{m}, h_{\Lambda}^{a}$ and the possible fluxes in the type $I I B$ string with $D 7$-branes. The latter is compactified on the orbifold $X_{6}=T^{4} / \mathbf{Z}_{2} \times T^{2}$ supplemented with the orientifold projection $\Omega(-1)^{F_{L}} I_{2}^{3}$. For the latter model the 3 -form flux $G_{3}$ is expressed as linear combination of the integral cohomology elements 17 $H^{3}\left(T^{4} / \mathbf{Z}_{2} \times T^{2}\right):$

$$
\begin{aligned}
\frac{1}{(2 \pi)^{2} \alpha^{\prime}} G_{3} & =\sum_{i=0}^{3}\left[\left(a^{i}-S c^{i}\right) \alpha_{i}+\left(b_{i}-S d_{i}\right) \beta^{i}\right] \\
& =\sum_{i=0}^{3}\left(A^{i} \omega_{A_{i}}+B^{i} \omega_{B_{i}}\right) .
\end{aligned}
$$

The complex 3-form flux is defined as follows: $G_{3}=F_{3}-S H_{3}$, where $F_{3}=d C_{2}$ comes from the Ramond sector and $H_{3}=d B_{2}$ comes from the Neveu-Schwarz sector. While in the complex basis the Hodge-decomposition $H^{3}=H^{(3,0)} \oplus H^{(2,1)} \oplus$ $H^{(1,2)} \oplus H^{(0,3)}$ of $G_{3}$ is manifest, the $S L(2, \mathbf{Z})_{S}$-invariance of $G_{3}$ is manifest in the real basis.

Without D3- and D7-branes, i.e. in the absence of open string moduli and 2-form world-volume fluxes on the $D 7$-branes, the superpotential originates from the closed string sector only and is given by (1.1):

$$
W=\frac{1}{(2 \pi)^{2} \alpha^{\prime}} \int_{X_{6}} G_{3} \wedge \Omega
$$

17 See appendix C for more details. 
with $\Omega$ the holomorphic three-form of $X_{6}$, i.e. $\Omega=d z^{1} \wedge d z^{2} \wedge d z^{3}$. This is to be compared with type IIB compactified on a Calabi-Yau manifold leading to an $\mathrm{N}=2$ closed string sector. It only depends on the dilaton $S$ and complex structure moduli $U^{j}$. With the definitions of appendix C, we obtain for (5.43) the following expression [20]:

$$
\begin{aligned}
W_{1}\left(S, U^{1}, U^{2}, U^{3}\right) & =-\left(a^{1}-S c^{1}\right) U^{2} U^{3}-\left(a^{2}-S c^{2}\right) U^{1} U^{3}-\left(a^{3}-S c^{3}\right) U^{1} U^{2} \\
& -\left(b_{0}-S d_{0}\right)+\left(a^{0}-S c^{0}\right) U^{1} U^{2} U^{3}-\sum_{i=1}^{3}\left(b_{i}-S d_{i}\right) U^{i}
\end{aligned}
$$

On the other hand, in the expression (5.41) from gauged supergravity, that piece (5.44) is (for $C^{7, a}=0$ ) captured by the sum $\Lambda=0, \ldots, 3$, provided the following identifications are made:

$$
\begin{aligned}
& \sqrt{2} a^{0}=-f_{1}^{2}-f_{3}^{2}+h_{1}^{2}+h_{3}^{2} \quad, \quad \sqrt{2} a^{1}=f_{1}^{1}+f_{3}^{1}-h_{1}^{1}-h_{3}^{1}, \\
& \sqrt{2} a^{2}=f_{1}^{1}+f_{3}^{1}+h_{1}^{1}+h_{3}^{1} \quad, \quad \sqrt{2} a^{3}=-f_{0}^{2}+f_{2}^{2}+h_{0}^{2}-h_{2}^{2}, \\
& \sqrt{2} b_{0}=f_{0}^{2}-f_{2}^{2}+h_{0}^{2}-h_{2}^{2} \quad, \quad \sqrt{2} b_{1}=-f_{0}^{1}+f_{2}^{1}-h_{0}^{1}+h_{2}^{1}, \\
& \sqrt{2} b_{2}=-f_{0}^{1}+f_{2}^{1}+h_{0}^{1}-h_{2}^{1} \quad, \quad \sqrt{2} b_{3}=-f_{1}^{2}-f_{3}^{2}-h_{1}^{2}-h_{3}^{2}, \\
& \sqrt{2} c^{0}=f_{0}^{2}+f_{2}^{2}-h_{0}^{2}-h_{2}^{2} \quad, \quad \sqrt{2} c^{1}=-f_{0}^{1}-f_{2}^{1}+h_{0}^{1}+h_{2}^{1}, \\
& \sqrt{2} c^{2}=-f_{0}^{1}-f_{2}^{1}-h_{0}^{1}-h_{2}^{1} \quad, \quad \sqrt{2} c^{3}=-f_{1}^{2}+f_{3}^{2}+h_{1}^{2}-h_{3}^{2}, \\
& \sqrt{2} d_{0}=f_{1}^{2}-f_{3}^{2}+h_{1}^{2}-h_{3}^{2} \quad, \quad \sqrt{2} d_{1}=-f_{1}^{1}+f_{3}^{1}-h_{1}^{1}+h_{3}^{1}, \\
& \sqrt{2} d_{2}=-f_{1}^{1}+f_{3}^{1}+h_{1}^{1}-h_{3}^{1} \quad, \quad \sqrt{2} d_{3}=f_{0}^{2}+f_{2}^{2}+h_{0}^{2}+h_{2}^{2} .
\end{aligned}
$$

This gives an one-to-one map 18 of the sixteen flux-components $a^{i}, b_{i}, c^{i}, d_{i}$ appearing in the expansion (5.42) and the sixteen gaugings $f_{\Lambda}^{m}, h_{\Lambda}^{a}, \Lambda=0, \ldots, 3, a, m=$ 1,2 .

Note, that in addition, there could be a dependence of the closed string superpotential on the modulus $T$ accounting for the $K 3$-volume. The latter originates from the sections $F_{\Lambda}$.

Let us now discuss the dependence of the superpotential on the open string moduli $C^{7, a}$. The quadratic piece $C^{7, a}$ in the sections $X^{0}, X^{2}$ appears as a matter of the symmetries of the $\mathrm{N}=2$ prepotential (5.11). Eventually, as we shall see in the next section, these terms provide supersymmetric mass terms for the D7-brane fields $C^{7, a}$ describing the transverse position along the torus $T^{2,3}$. Therefore, let

18 This is the restriction of the map in Eqs. (4.12) (4.13) to the orbifold. Alternatively, the 
us move on to that part in (5.41), which stems from the sum $\Lambda=4, \ldots, 4+n_{7}$ or $a=1, \ldots, n_{7}$ :

$$
\begin{aligned}
W_{2}\left(C^{7, a}, U^{1}, U^{2}\right)=\sum_{a=1}^{n_{7}} C^{7, a}\{ & f_{a}^{1}\left(U^{1}+U^{2}\right)-f_{a}^{2}\left(1-U^{1} U^{2}\right) \\
& \left.+h_{a}^{1}\left(U^{1}-U^{2}\right)-h_{a}^{2}\left(1+U^{1} U^{2}\right)\right\} .
\end{aligned}
$$

As discussed around Eq. (4.13), these gaugings are related to world-volume 2-form fluxes on the $D 7$-brane. The latter is wrapped around the $T^{4} / \mathbf{Z}_{2}$ orbifold. Hence, let us briefly introduce the 2-form cohomology of this orbifold.

The toroidal orbifold $T^{4} / \mathbf{Z}_{2}$ is the simplest of the orbifold limits of $K 3$. The $\mathbf{Z}_{2}-$ action $\left(z_{1}, z_{2}\right) \rightarrow\left(-z_{1},-z_{2}\right)$ has 16 fixed points, namely $(0,0),\left(0, \frac{1}{2}\right),\left(0, \frac{1}{2} i\right),\left(0, \frac{1}{2}+\right.$ $\left.\frac{1}{2} i\right),\left(\frac{1}{2}, 0\right), \ldots,\left(\frac{1}{2}+\frac{1}{2} i, \frac{1}{2}+\frac{1}{2} i\right)$. We know to have $h^{(2,0)}+h^{(1,1)}+h^{(0,2)}=1+20+1$ two-forms on $T^{4} / \mathbf{Z}_{2}$. The first six of them are the following:

$$
\begin{array}{ll}
d z^{1} \wedge d z^{2}, & d \bar{z}^{1} \wedge d \bar{z}^{2} \\
d z^{1} \wedge d \bar{z}^{2}, & d \bar{z}^{1} \wedge d z^{2} \\
d z^{1} \wedge d \bar{z}^{1}, & d z^{2} \wedge d \bar{z}^{2}
\end{array}
$$

The remaining $16(1,1)$-forms correspond to the collapsed 2-cycles at the orbifold fixed points. We choose the complex structure

$$
\omega=d z^{1} \wedge d z^{2}
$$

inverse relations are:

$$
\begin{array}{lll}
f_{0}^{1}=-\frac{\sqrt{2}}{4}\left(b_{1}+b_{2}+c^{1}+c^{2}\right) \quad, \quad f_{1}^{1}=\frac{\sqrt{2}}{4}\left(a^{1}+a^{2}-d_{1}-d_{2}\right) \quad, \quad f_{2}^{1}=\frac{\sqrt{2}}{4}\left(b_{1}+b_{2}-c^{1}-c^{2}\right) \\
f_{3}^{1}=\frac{\sqrt{2}}{4}\left(a^{1}+a^{2}+d_{1}+d_{2}\right) \quad, \quad f_{0}^{2}=\frac{\sqrt{2}}{4}\left(-a^{3}+b_{0}+c^{0}+d_{3}\right) \quad, \quad f_{1}^{2}=-\frac{\sqrt{2}}{4}\left(a^{0}+b_{3}+c^{3}-d_{0}\right) \\
f_{2}^{2}=\frac{\sqrt{2}}{4}\left(a^{3}-b_{0}+c^{0}+d_{3}\right) \quad, \quad f_{3}^{2}=-\frac{\sqrt{2}}{4}\left(a^{0}+b_{3}-c^{3}+d_{0}\right), \quad h_{0}^{1}=\frac{\sqrt{2}}{4}\left(-b_{1}+b_{2}+c^{1}-c^{2}\right), \\
h_{1}^{1}=\frac{\sqrt{2}}{4}\left(-a^{1}+a^{2}-d_{1}+d_{2}\right) \quad, \quad h_{2}^{1}=\frac{\sqrt{2}}{4}\left(b_{1}-b_{2}+c^{1}-c^{2}\right) \quad, \quad h_{3}^{1}=\frac{\sqrt{2}}{4}\left(-a^{1}+a^{2}+d_{1}-d_{2}\right) \\
h_{0}^{2}=\frac{\sqrt{2}}{4}\left(a^{3}+b_{0}-c^{0}+d_{3}\right) \quad, \quad h_{1}^{2}=\frac{\sqrt{2}}{4}\left(a^{0}-b_{3}+c^{3}+d_{0}\right) \quad, \quad h_{2}^{2}=-\frac{\sqrt{2}}{4}\left(a^{3}+b_{0}+c^{0}-d_{3}\right), \\
h_{3}^{2}=-\frac{\sqrt{2}}{4}\left(-a^{0}+b_{3}+c^{3}+d_{0}\right) .
\end{array}
$$


which may be decomposed w.r.t to the real cohomology $19\left(z^{j}=x^{j}+U^{j} y^{j}, j=\right.$ $1,2)$

$$
\begin{array}{ll}
\alpha_{0}=d x^{1} \wedge d x^{2} & , \quad \beta^{0}=d y^{1} \wedge d y^{2}, \\
\alpha_{1}=d y^{1} \wedge d x^{2} & , \quad \beta^{1}=-d x^{1} \wedge d y^{2}, \\
\alpha_{2}=d x^{1} \wedge d y^{1} & , \quad \beta^{2}=-d x^{2} \wedge d y^{2}
\end{array}
$$

with intersection matrix $M_{i}^{j}=\int_{T^{4} / Z_{2}} \alpha_{i} \wedge \beta^{j}=\delta_{i}^{j}$, according to

$$
\omega_{0}=\alpha_{0}+\alpha_{1} U^{1}-\beta^{1} U^{2}+\beta^{0} U^{1} U^{2}=\left.\sum_{I} \Pi^{I} \eta_{I}\right|_{T^{2} \times T^{2} / \mathbf{z}_{2}}
$$

As indicated, this is the expansion of $\omega$ in terms of the periods $\Pi^{I}$ in the notation of (2.4), restricted to the subset $S O(2,2) \subset S O(3,19)$. The Kähler form is chosen to be:

$$
j_{0}=\sum_{j=1}^{2} \operatorname{Im} \mathcal{T}^{j} d x^{j} \wedge d y^{j}
$$

which is again a restriction of the general expansion $j=\Pi^{I, 3} \eta_{I}$, to a 2 real dimensional subspace. The moduli $\mathcal{T}^{j}$ are the Kähler moduli in the string basis, see $E q$. (5.20). The most general 2 -form flux $\mathcal{F}$ on the $D 7$-brane world-volume, which is wrapped on $T^{4} / \mathbf{Z}_{2}$, has six non-vanishing components $F_{i j}^{a}$ referring to the integral cohomology $H^{2}\left(T^{4}, \mathbf{Z}\right)$ elements:

$$
\begin{aligned}
\mathcal{F}^{a} & =\sum_{i j} \mathcal{F}_{i j}^{a}=\mathcal{F}_{N P}^{a}+\mathcal{F}_{P}^{a}, \\
\mathcal{F}_{N P}^{a} & =2 \pi \alpha^{\prime}\left(F_{12}^{a} \alpha_{2}-F_{34}^{a} \beta^{2}\right), \\
\mathcal{F}_{P}^{a} & =2 \pi \alpha^{\prime} \quad\left(F_{13}^{a} \alpha_{0}+F_{23}^{a} \alpha_{1}-F_{14}^{a} \beta^{1}+F_{24}^{a} \beta^{0}\right) .
\end{aligned}
$$

We have split off a piece $\mathcal{F}_{N P}^{a}\left(\right.$ i.e. $\left.j_{0} \wedge \mathcal{F}_{N P}^{a} \neq 0\right)$ of the cohomology $H^{1,1}\left(T^{4}, \mathbf{Z}\right)$, which is generically non-primitive in the restricted Kähler structure $j_{0}$ of $E q$. (5.53). On the other hand, the part $\mathcal{F}_{P}^{a}$ is always primitive in the same Kähler structure (i.e. $\left.j_{0} \wedge \mathcal{F}_{P}^{a}=0\right)$. The flux components $F_{i j}^{a}$ obey the quantization rule: $F_{i j}^{a}=2 \pi \frac{n_{i j}^{a}}{m_{i j}^{a}}$.

19 Instead, we may also choose the basis

$$
\begin{aligned}
& e_{1}=\alpha_{0}-\beta^{0}, e_{2}=\alpha_{1}-\beta^{1}, e_{3}=\alpha_{2}-\beta^{2}, \\
& e_{4}=\alpha_{0}+\beta^{0}, e_{5}=-\alpha_{1}-\beta^{1}, e_{6}=\alpha_{2}+\beta^{2},
\end{aligned}
$$

which shares the intersection properties of $S O(3,3)$, i.e. $\left(e_{i}, e_{i}\right)=-2, i=1,2,3,\left(e_{i}, e_{i}\right)=$ $2, i=4,5,6$, and $\left(e_{i}, e_{j}\right)=0, i \neq j$. 
In the $F$-theory compactification on $X_{4}=X_{H} \times \widetilde{X}_{V}$, the 2 -form flux $\mathcal{F}^{a}$ arises from the piece

$$
G_{4}=\sum_{a} \mathcal{F}^{a} \wedge \widetilde{\eta}_{a+4}
$$

with $\widetilde{\eta}_{a+4}$ the $4 \times 42$-forms on the exceptional spheres of the $4 D_{4}$ singularities. This flux induces the two potentials $W$ and $\breve{W}$ in (3.1), associated with a four-dimensional $F$-term and $D$-term, respectively. On the $a$-th $D 7$-brane, the flux integrals on the "lower" K3 become

$$
\begin{aligned}
\left.W\right|_{D 7^{a}} & : \int_{X_{H}} \mathcal{F}_{P}^{a} \wedge \omega_{0}=F_{24}^{a}-F_{14}^{a} U^{1}-F_{23}^{a} U^{2}+F_{13}^{a} U^{1} U^{2}, \\
\left.d \breve{W}\right|_{D 7^{a}}: & \int_{X_{H}} \mathcal{F}_{N P}^{a} \wedge j_{0}=\operatorname{Im} \mathcal{T}^{2} F_{12}^{a}+\operatorname{Im} \mathcal{T}^{1} F_{34}^{a} .
\end{aligned}
$$

Thus the "non-primitive" part $\mathcal{F}_{N P}$ of the 2 -form flux gives rise to a $D$-term potential for the moduli $\mathcal{T}^{j}$ in (5.53), which vanishes if the condition (5.12) is met and $\mathcal{F}_{N P}^{a}$ is actually primitive at this point in the moduli. The primitive part $\mathcal{F}_{P}$ gives rise to an $F$-term superpotential $W$ for the moduli $U^{1}, U^{2}$. Moreover, the latter precisely reproduces the piece (5.47) of the superpotential, derived from gauged supergravity. Hence, we recover the dictionary (4.13) for the case of the orbifold 20 , namely the supergravity gaugings $f_{a}^{m}, h_{a}^{m}$, correspond to the flux-components as:

$$
\begin{array}{ll}
f_{a}^{1}=-\frac{1}{2}\left(F_{14}^{a}+F_{23}^{a}\right), & h_{a}^{1}=-\frac{1}{2}\left(F_{14}^{a}-F_{23}^{a}\right), \\
f_{a}^{2}=-\frac{1}{2}\left(F_{24}^{a}-F_{13}^{a}\right), & h_{a}^{2}=-\frac{1}{2}\left(F_{24}^{a}+F_{13}^{a}\right), \\
f_{a}^{3}=\frac{1}{2}\left(F_{12}^{a}+F_{34}^{a}\right), & h_{a}^{3}=-\frac{1}{2}\left(F_{12}^{a}-F_{34}^{a}\right) .
\end{array}
$$

From Eqs. (5.56) we see, that the flux components $F_{12}^{a}, F_{34}^{a}$ give rise to a $D$-term potential, which fixes some of the Kähler moduli ( $c f . E q$. (5.13)). On the other hand, the remaining components $F_{13}^{a}, F_{14}^{a}, F_{23}^{a}, F_{24}^{a}$ correspond to the gaugings $f_{a}^{1,2}, h_{a}^{1,2}$ and enter the $F$-term superpotential. Eventually, the latter fix some of the complex structure moduli, as it has been recently also found in Ref. [36]. To conclude, we have found the precise mapping of supergravity gaugings $f_{\Lambda}^{m}, h_{\Lambda}^{a}$ and fluxes in type $I I B$ string theory for the $T^{4} / \mathbf{Z}_{2} \times T^{2}$ orientifold, for $\Lambda=0,1, \ldots, 4+n_{7}$.

From our discussion of $F$ - and $D$-terms in sect. 3.1 it follows that this is not the full story. Since we are studying minima of a potential on the moduli space, we

20 Note, that the gaugings $f_{\Lambda}^{m}, h_{\Lambda}^{a}$ refer to the lattice $S O(3,3)$, i.e. the basis $e_{i}$ given in the last footnote. This is way we obtain linear combinations as relations, which reflect the change of basis from $\alpha_{i}, \beta^{j}$ to $e_{i}$. 
can not simply restrict to the particular subset (5.52) and (5.53) of moduli. Instead one has to study the full potential for all K3 moduli, including the ten geometrical untwisted moduli as well as twisted moduli. In particular the flux $\mathcal{F}_{N P}^{a}$ gives a contribution to the $F$-term potential $W$ and the $D$ term potential $d \breve{W}$ in these moduli and similarly the flux $\mathcal{F}_{P}^{a}$ contributes to both potentials. The restriction to the subset parametrized by $U^{i}$ and $T^{i}$ is consistent, if the minimization of the full potential allows to eliminate all these extra moduli from the potential.21 In this case the truncated potential (5.56) can be interpreted as the result of the minimization of the full potential in all moduli except $T^{i}, U^{i}$, and the subsequent minimization of the moduli $T^{i}, U^{i}$ can then be studied using the truncated potentials.

According to the classification of complex structure preserving fluxes in section 3.1, the 2-form flux $\mathcal{F}_{N P}^{a}$ fulfills the above consistency criterion, but the ansatz for $\mathcal{F}_{P}^{a}$ does not. Thus one can not fix complex structure moduli with this flux in the orientifold/orbifold. As described in section 3.4, there is a different set of 2-form fluxes which yield a potential that does have a minimum on the restricted orbifold moduli space parametrized by $T^{i}, U^{i}$ and therefore can be consistently incorporated in the orbifold model. These fluxes couple to the off-diagonal and twisted moduli of the orbifold. On the other hand, the only way to fix the complex structure moduli within the simple orientifold/orbifold model based on the coset $S O(2,2) \times S O(1,1)$ is to use the bulk 3 -form fluxes. Fixing the complex structure with bulk 3 -form flux, this leaves an 18 dimensional moduli space of Kähler moduli which can not be consistently fixed within the orbifold model based on $S O(2,2) \times S O(1,1)$.

Due to the block-diagonal form of $e$ ( $c f . E q$. (5.34)), independent on the choice of gaugings $f_{\Lambda}^{m}, h_{\Lambda}^{a}$, besides the vectormultiplet moduli $S, T, U, C^{7}, C^{3}$, only the $S O(2,2)$-part of $e$, i.e. the complex structure moduli $U^{1}, U^{2}$ of $T^{4} / \mathbf{Z}_{2}$ may enter the superpotential $W$ through the Killing potentials $P^{1}, P^{2}$. Hence, quite generically we obtain a superpotential $W=W\left(S, U^{1}, U^{2}, U^{3}, C^{7, a}, C^{3, a}\right)$ depending on the fields $S, U^{j}, C^{7, a}, C^{3, a}$. This superpotential $W$, given in $E q$. (5.39), gives rise to the $F$-term potential

$$
V_{F}=e^{K_{V}+K_{H}}\left(g^{i \bar{j}} D_{i} W D_{\bar{j}} \bar{W}-3|W|^{2}\right),
$$

with the index $i$ running over both the vectormultiplet moduli $S, T, U, C^{7}, C^{3}$ and the hypermultiplet moduli $U^{1}, U^{2}, T^{1}, T^{2}$. Since the superpotential does not depend on the Kähler moduli, the scalar potential $V_{F}$ does not depend on them either as

21 This happens if the extra moduli are either fixed to constant values at the minimum, or some of the moduli decouple at the minimum and remain true moduli. 
a result of no-scale structure. In addition, there is a $D$-term scalar potential $V_{D}$, derived from the Killing potential $P^{3}$, given in $E q$. (5.38)

$$
\begin{aligned}
V_{D}=g_{D_{7}}^{2} \cdot\left|P^{3}\right|^{2} & \sim\left|\frac{1}{2}\left(\frac{1}{\sqrt{\rho}}+\sqrt{\rho}\right) f_{\Lambda}^{3}+\frac{1}{2}\left(\frac{1}{\sqrt{\rho}}-\sqrt{\rho}\right) h_{\Lambda}^{3}\right|^{2}, \\
& \sim\left|\left(f_{\Lambda}^{3}+h_{\Lambda}^{3}\right) \operatorname{Im} \mathcal{T}^{1}+\left(f_{\Lambda}^{3}-h_{\Lambda}^{3}\right) \operatorname{Im} \mathcal{T}^{2}\right|^{2}
\end{aligned}
$$

in agreement with Eq. (3.19). The above equations depends on the two Kähler moduli in (5.53). The term $\sim f_{\Lambda}^{3}$ is the universal $D$-term $j_{0} \wedge j_{0}$ for the orbifold, which drives the theory to the boundary of the moduli. On the other hand, the case $h_{\Lambda}^{3} \neq 0, \Lambda>3$, gauges a $U(1)$ symmetry of $D 7$-brane vectors and gives rise to a non-vanishing $D$-term potential of the type (3.7), which is minimized for

$\rho \equiv \frac{T_{2}^{1}}{T_{2}^{2}}=1$. Comparing with Eqs. (5.13) and (5.56), this gauging may be identified with turning on the world-volume 2 -fluxes $F_{12}^{a}, F_{34}^{a}$ on $D 7$-branes.

Note, as pointed out already in [23,24], the condition $e=0$, i.e. $L=1$ generically fixes all hypermultiplet moduli, except the volume $e^{\Phi}=\sqrt{T_{2}^{1} T_{2}^{2}}$, to

$$
\rho=1 \quad, \quad\left|U^{1}\right|^{2}=\left|U^{2}\right|^{2}=1 .
$$

\section{The $\mu$-term and soft SUSY breaking terms in the type $I I B K 3 \times T^{2}$ orientifold}

In this section we study the $D=4$ effective $\mathrm{N}=1$ supergravity action with spontaneous supersymmetry breaking due to non-vanishing auxiliary $F$-term components of the moduli fields. In this framework we calculate the flux-induced soft supersymmetry breaking terms of the effective four-dimensional $D 7$-brane gauge theory in lines of 37 .

Compared to the effective approach performed in Refs. 220,22, the main difference is the superpotential (5.41) depending now also on the open string moduli $C^{7, a}$ and $C^{3, b}$ referring to the $D 7$ - and $D 3$-brane positions along the torus $T^{2}$. In particular, there is a supersymmetric mass term for the $D 7$-brane moduli $C^{7, a}$ in the superpotential. The latter has been argued to be present by considering $F-$ theory compactified on $K 3 \times K 3$ in Ref. [10] or after dimensional reduction of the Born-Infeld action of a single D7-brane in Ref. [21]. The dependence of the mass 
$m$ of the $D 7$-brane modulus $C^{7}$ on the $(0,3)$ and $(1,2)$ flux-components has been found to be [20,22]

$m^{2} \sim \frac{1}{Y} \frac{1}{(S-\bar{S})\left(U^{3}-\bar{U}^{3}\right)}\left\{\left|\int G_{3} \wedge \Omega\right|^{2}+\left|\int G_{3} \wedge \omega_{B_{1}}\right|^{2}+\left|\int G_{3} \wedge \omega_{B_{2}}\right|^{2}\right\}$

with:

$$
Y=(S-\bar{S}) \prod_{j=1}^{3}\left(T^{j}-\bar{T}^{j}\right)\left(U^{j}-\bar{U}^{j}\right)
$$

Due to the presence of the supersymmetric mass term, depending linearly on the gaugings $f_{0}^{m}, h_{0}^{a}$ and $f_{2}^{m}, h_{2}^{a}$, we expect an additional correction to the $D 7$-brane scalar mass. This correction represents a supersymmetric mass term and is important to give a mass also to the fermionic partners of those moduli.

\subsection{Calculating the soft terms for D7-branes}

We first consider the case with just D7-branes (no D3-branes), no 2-form fluxes and set the $f_{\Lambda}^{m}$ and the $h_{\Lambda}^{a}$ to zero for $\Lambda=4, \ldots$, such that we are only left with the gaugings $f_{\Lambda}^{m}, h_{\Lambda}^{a}$ with $\Lambda=0, \ldots, 3$. The precise mapping of these gaugings to the 3 -form flux components has been given in the previous section.

This case fits nicely into the formalism of [37]. The soft terms are obtained by Taylor expanding the scalar potential around $C^{7 a}=\bar{C}^{7 a}=0$, i.e. in the following the $C^{7 a}, \bar{C}^{7 a}$ are assumed to be small.

First, we will introduce some notation in order to make the results look less cluttered. We want to cast our Kähler- and superpotential into the following form:

$$
\begin{aligned}
K & =K_{V}+K_{H}=\widehat{K}+G_{C^{7 a} \bar{C}^{7 a}} C^{7 a} \bar{C}^{7 a}+\left(\frac{1}{2} H_{C^{7 a} C^{7 a}} C^{7 a} C^{7 a}+\text { h.c. }\right)+\ldots \\
W & =\widehat{W}+\frac{1}{2} \widetilde{\mu}_{C^{7 a} C^{7 a}} C^{7 a} C^{7 a}+\frac{1}{3} \widetilde{Y}_{A B C} C^{A} C^{B} C^{C}+\ldots
\end{aligned}
$$

Here, $\widehat{K}$ and $\widehat{W}$ refer to the respective potentials in the bulk and have the following form:

$$
\begin{aligned}
\widehat{K} & =-\sum_{M \in\left\{S, T^{i}, U^{i}\right\}} \ln (M-\bar{M})=-\ln Y, \\
\widehat{W} & =\frac{1}{\sqrt{2}}\left\{(1-S U) A_{0}-(S+U) A_{1}-(1+S U) A_{2}-(U-S) A_{3}\right\} .
\end{aligned}
$$


Here we introduced the shorthand

$$
A_{i}=f_{i}^{1}\left(U^{1}+U^{2}\right)-f_{i}^{2}\left(1-U^{1} U^{2}\right)+h_{i}^{1}\left(U^{1}-U^{2}\right)-h_{i}^{2}\left(1+U^{1} U^{2}\right) .
$$

The two functions $\widehat{K}$ and $\widehat{W}$ depend on the closed string moduli fields, only. To be able to write $K=K_{V}+K_{H}$ in the form (6.3), we expand the logarithm for small $C$. We then get:

$$
G_{C^{7 a} \bar{C}^{7 a}}=\frac{-1}{(S-\bar{S})(U-\bar{U})}, \quad H_{C^{7 a} C^{7 a}}=\frac{1}{(S-\bar{S})(U-\bar{U})}=-G_{C^{7 a} \bar{C}^{7 a}} .
$$

The expression for $\widetilde{\mu}_{C^{7 a} C^{7 a}}$ can be read off directly from the superpotential (5.41):

$$
\widetilde{\mu}_{C^{7 a} C^{7 a}}=\frac{1}{\sqrt{2}}\left[A_{0}+A_{2}\right] .
$$

The remaining part of $W$ is zero in the case of only $D 7$-branes and no $D 3$-branes.

The scalar potential $V$ may be determined with the formula (5.58):

$$
V=e^{K}\left[G^{I \bar{J}}\left(D_{I} W\right)(D \bar{J} \bar{W})-3|W|^{2}\right],
$$

with the Kähler covariant derivative $D_{I} W=\partial_{I} W+W \partial_{I} K$ and the sum running over all moduli $S, T^{j}, U^{j}, C^{7, a}$. The expansion of (6.8) w.r.t. the matter field modulus $\phi \equiv C$ up to third order may be arranged according to (cf. Refs. [37]):

$$
\begin{aligned}
V & =\widehat{V}(M, \bar{M})+\frac{1}{2} D^{2}+G^{C^{7 a} \bar{C}^{7 a}} \frac{\partial W^{e f f}}{\partial C^{7 a}} \frac{\partial \bar{W}^{e f f}}{\partial \bar{C}^{7 a}} \\
& +m_{C^{7 a} \bar{C}^{7 a}}^{2}\left|C^{7 a}\right|^{2}+\frac{1}{3} A_{A B C} C^{A} C^{B} C^{C}+\frac{1}{2} B_{C^{7 a} C^{7 a}}\left(C^{7 a}\right)^{2}+\ldots
\end{aligned}
$$

The second and third terms of the first line preserve supersymmetry and $W^{e f f}$ may be considered as an effective $\mathrm{N}=1$ superpotential, which may take the following form:

$$
W^{e f f}=\frac{1}{2} \mu_{C^{7 a} C^{7 a}}\left(C^{7 a}\right)^{2}+\frac{1}{3} Y_{A B C} C^{A} C^{B} C^{C}+\ldots .
$$

Note that, as we shall see later, the quantities $\mu$ and $Y$ are not holomorphic. Note especially that in the case of only $D 7$-branes, all being transverse to the torus $T^{2,3}$, the three-point functions between the matter fields $C^{7, a}$ are zero, i.e. $0=\widetilde{Y}_{A B C}=$ $e^{-\widehat{K} / 2} Y_{A B C}$. From now on, we will for the sake of clearer notation suppress the superscript $a$ labeling the brane.

The first thing we need to calculate on the road to the soft terms are the $F$-terms. They can be written down in a very compact way as follows:

$$
\bar{F}^{\bar{N}}=e^{\widehat{K} / 2} \widehat{K}^{\bar{N} L}\left(\partial_{L} \widehat{W}+\widehat{W} \partial_{L} \widehat{K}\right)=Y^{-1 / 2}(N-\bar{N})^{2} D_{N} \widehat{W}
$$


As an illustrative example, we display the expression for $\bar{F}^{\bar{S}}$ :

$$
\bar{F}^{\bar{S}}=Y^{-1 / 2}(S-\bar{S}) \frac{1}{\sqrt{2}}\left\{(1-\bar{S} U) A_{0}-(\bar{S}+U) A_{1}-(1+\bar{S} U) A_{2}-(U-\bar{S}) A_{3}\right\} .
$$

Because of the exact correspondence between the superpotential from the gaugings and the flux superpotential $\int G_{3} \wedge \Omega$, we can express everything through flux integrals:

$$
\widehat{W}=\int G_{3} \wedge \Omega, \quad D_{S} \widehat{W} \propto \int \bar{G}_{3} \wedge \Omega, \quad D_{U^{i}} \widehat{W} \propto \int G_{3} \wedge \omega_{A_{i}} .
$$

Together with

$$
m_{3 / 2}=Y^{-1 / 2} \widehat{W}
$$

we can now write down the expression for the bulk scalar potential. In the no-scale case, the cosmological constant $\widehat{V}(M, \bar{M})$ consists of the $F$-terms of the dilaton and complex structure moduli, only:

$$
\begin{aligned}
\widehat{V}(M, \bar{M}) & =\widehat{K}_{S \bar{S}} F^{S} \bar{F}^{\bar{S}}+\sum_{j=1}^{3} \widehat{K}_{U^{j} \bar{U}^{j}} F^{U^{j}} \bar{F}^{j} \\
& =|Y|^{-1}\left\{\left|\int \bar{G}_{3} \wedge \Omega\right|^{2}+\sum_{i=1}^{3}\left|\int G_{3} \wedge \omega_{A_{i}}\right|^{2}\right\} .
\end{aligned}
$$

Before we can write down the scalar mass term, we must think about the curvature tensor, which is very simple in this case:

$$
R_{S \bar{S} 3 \overline{3}}=\frac{-1}{(S-\bar{S})^{2}} G_{C^{7} \bar{C}^{7}}, \quad R_{U \bar{U} a \bar{a}}=\frac{-1}{(U-\bar{U})^{2}} G_{C^{7} \bar{C}^{7}} .
$$

All other components are zero. So the scalar masses become

$$
\begin{aligned}
m_{C^{7} \bar{C}^{7}, \text { soft }}^{2} & =\left(\left|m_{3 / 2}\right|^{2}+\widehat{V}\right) G_{C^{7} \bar{C}^{7}-\bar{F}^{M} F^{\bar{N}} R_{M \bar{N} 3 \overline{3}}} \\
& =|Y|^{-1}\left\{\left|\int G_{3} \wedge \Omega\right|^{2}+\left|\int G_{3} \wedge \omega_{A_{1}}\right|^{2}+\left|\int G_{3} \wedge \omega_{A_{2}}\right|^{2}\right\} G_{C^{7} \bar{C}^{7}},
\end{aligned}
$$

which agrees with the form given in (6.1).

Let us now determine the supersymmetric mass term $\mu_{C^{7} C^{7}}$, which gives the mass $m_{C^{7} C^{7}}^{f}=\partial_{C^{7}}^{2} W^{e f f}=\mu_{C^{7} C^{7}}$ to the fermionic partners of the D7-brane moduli $C^{7}$. The $\mu$-term has the following form:

$$
\begin{aligned}
\mu_{C^{7} C^{7}} & =e^{\widehat{K} / 2} \widetilde{\mu}_{C^{7} C^{7}}+m_{3 / 2} H_{C^{7} C^{7}}-\bar{F}^{\bar{M}} \bar{\partial}_{\bar{M}} H_{C^{7} C^{7}} \\
& =-\frac{Y^{-1 / 2}}{\sqrt{2}} G_{C^{7} \bar{C}^{7}}\left[(1-\overline{S U}) A_{0}-(\bar{S}+\bar{U}) A_{1}-(1+\overline{S U}) A_{2}-(\bar{U}-\bar{S}) A_{3}\right] .
\end{aligned}
$$


In lines of the Giudice-Masiero effect [38], in the above equation the $\widetilde{\mu}$-term of (6.3) combines with supersymmetry breaking terms into an effective $\mu$-term in (6.10). The latter gives a mass to the non-chiral fermions of the $D 7$-brane moduli superfields.

Naturally, we are now interested in learning which of the flux components contribute to $\mu$. We find that

$$
\mu_{C^{7} C^{7}}=-Y^{-1 / 2} G_{C^{7} \bar{C}^{7}} \int \bar{G}_{3} \wedge \omega_{A_{3}} .
$$

This means that only a $(2,1)$-flux component (which preserves supersymmetry) contributes to $\mu$-term.

From the expansion of (6.8) we find an additional (supersymmetric) contribution to $m_{C^{7} \bar{C}^{7}}^{2}$. This mass comes from $G^{C^{7} \bar{C}^{7}} \frac{\partial W^{e f f}}{\partial C^{7}} \frac{\partial \bar{W}^{e f f}}{\partial \bar{C}^{7}}$, after extracting the order $\left|C^{7}\right|^{2}$

$$
\begin{aligned}
m_{C^{7} \bar{C}^{7}, \text { Susy }}^{2} & =\frac{i}{Y(S-\bar{S})\left(U^{3}-\bar{U}^{3}\right)} \\
& \times\left|(S-\bar{S})\left(U^{3}-\bar{U}^{3}\right) \widetilde{\mu}_{C^{7} C^{7}}-\widehat{W}+(S-\bar{S}) \frac{\partial \widehat{W}}{\partial S}+\left(U^{3}-\bar{U}^{3}\right) \frac{\partial \widehat{W}}{\partial U^{3}}\right|^{2} \\
& =G^{C^{7} \bar{C}^{7}}\left|\mu_{C^{7} C^{7}}\right|^{2} .
\end{aligned}
$$

The total mass term therefore amounts to:

$m_{C^{7} \bar{C}^{7}, \text { soft+Susy }}^{2}=\frac{G_{C^{7} \bar{C}^{7}}}{|Y|}\left\{\left|\int G_{3} \wedge \Omega\right|^{2}+\left|\int G_{3} \wedge \omega_{A_{1}}\right|^{2}+\left|\int G_{3} \wedge \omega_{A_{2}}\right|^{2}+\left|\int \bar{G}_{3} \wedge \omega_{A_{3}}\right|^{2}\right\}$.

The trilinear couplings $A_{A B C}$ are zero in our case. The $B$-term is obtained via the expansion of the scalar potential as seen in (6.9). We find for $B$ :

$$
\begin{aligned}
\frac{1}{2} B_{C^{7} C^{7}}= & |Y|^{-1}\left\{H _ { C ^ { 7 } C ^ { 7 } } \left\{-\sum_{i=1}^{3}\left|\partial_{U^{i}} \widehat{W}\left(U^{i}-\bar{U}^{i}\right)\right|^{2}-\left|\partial_{S} \widehat{W}(S-\bar{S})\right|^{2}\right.\right. \\
& -4|\widehat{W}|^{2}+(S-\bar{S})(U-\bar{U})\left[\partial_{S} \widehat{W} \partial_{\bar{U}} \widehat{\bar{W}}+\partial_{U} \widehat{W} \partial_{\bar{S}} \widehat{\bar{W}}\right] \\
& \left.+\left[\widehat{\bar{W}}\left[\sum_{i=1}^{3} \partial_{U^{i}} \widehat{W}\left(U^{i}-\bar{U}^{i}\right)+\partial_{s} \widehat{W}(S-\bar{S})\right]+\text { h.c. }\right]\right\}-2 \widetilde{\mu} \widehat{\bar{W}} \\
& -\widetilde{\mu}\left[\partial_{\bar{U}^{1}} \widehat{\bar{W}}\left(U^{1}-\bar{U}^{1}\right)+\partial_{\bar{U}^{2}} \widehat{\widehat{W}}\left(U^{2}-\bar{U}^{2}\right)-\partial_{\bar{U}} \widehat{\bar{W}}(U-\bar{U})-\partial_{\bar{S}} \widehat{\bar{W}}(S-\bar{S})\right] \\
& +\partial_{U^{1}} \widetilde{\mu} \partial_{\bar{U}^{1}} \widehat{\bar{W}}\left(U^{1}-\bar{U}^{1}\right)^{2}+\partial_{U^{2}} \widetilde{\mu} \partial_{\bar{U}^{2}} \widehat{\widehat{W}}\left(U^{2}-\bar{U}^{2}\right)^{2} \\
& \left.+\widehat{\bar{W}}\left[\partial_{U^{1}} \widetilde{\mu}\left(U^{1}-\bar{U}^{1}\right)+\partial_{U^{2}} \widetilde{\mu}\left(U^{2}-\bar{U}^{2}\right)\right]\right\} .
\end{aligned}
$$


In order to express the result in terms of flux integrals, we must find the flux expressions for $\widetilde{\mu}$ and the $\partial_{U^{i}} \widetilde{\mu}$. It is important to realize that $\widetilde{\mu}=-\partial_{U} \partial_{S} \widehat{W}$. Writing $\widehat{W}=\int G_{3} \wedge \Omega$ with $G_{3}=F_{3}-S H_{3}$, we find after close examination

$$
\begin{aligned}
(S-\bar{S})(U-\bar{U}) \widetilde{\mu} & =-(S-\bar{S}) \int \partial_{S} G_{3} \wedge(U-\bar{U}) \partial_{U} \Omega \\
& =(S-\bar{S}) \int H_{3} \wedge\left(\Omega-\omega_{A_{3}}\right) \\
& =\int\left(\bar{G}_{3}-G_{3}\right) \wedge\left(\Omega-\omega_{A_{3}}\right) .
\end{aligned}
$$

So the $N S$-part of the flux is picked out by the $S$-derivative and the $(0,3),(3,0)$ and one of the $(1,2)$ and $(2,1)$-components are found to contribute to $\widetilde{\mu}$.

$(S-\bar{S})(U-\bar{U})\left(U^{i}-\bar{U}^{i}\right) \partial_{U^{i}} \widetilde{\mu}, i=1,2$ needs to be expressed in terms of flux integrals, as well. By similar reasoning as above, we find the following flux integrals:

$$
\begin{aligned}
& (S-\bar{S})(U-\bar{U})\left(U^{1}-\bar{U}^{1}\right) \partial_{U^{1}} \widetilde{\mu}=\int\left(\bar{G}_{3}-G_{3}\right) \wedge\left(\Omega-\omega_{A_{3}}-\omega_{A_{1}}+\omega_{B_{2}}\right), \\
& (S-\bar{S})(U-\bar{U})\left(U^{2}-\bar{U}^{2}\right) \partial_{U^{2}} \widetilde{\mu}=\int\left(\bar{G}_{3}-G_{3}\right) \wedge\left(\Omega-\omega_{A_{3}}-\omega_{A_{2}}+\omega_{B_{1}}\right) .
\end{aligned}
$$

Together with (6.12) we have now collected everything necessary to write (6.21) in terms of flux-integrals:

$$
\begin{aligned}
\frac{1}{2} B_{C^{7} C^{7}} & =|Y|^{-1} H_{C^{7} C^{7}}\left\{-\sum_{j=1}^{3} \int \bar{G}_{3} \wedge \omega_{A_{i}} \int \bar{G}_{3} \wedge \bar{\omega}_{A_{i}}-\int \bar{G}_{3} \wedge \Omega \int \bar{G}_{3} \wedge \bar{\Omega}\right. \\
& +\int\left(\bar{G}_{3}-G_{3}\right) \wedge \omega_{A_{3}} \int \bar{G}_{3} \wedge \bar{\Omega}+\int\left(\bar{G}_{3}-G_{3}\right) \wedge \bar{\Omega} \int \bar{G}_{3} \wedge \omega_{A_{3}} \\
& \left.+\int\left(\bar{G}_{3}-G_{3}\right) \wedge \bar{\omega}_{A_{2}} \int \bar{G}_{3} \wedge \bar{\omega}_{A_{1}}+\int\left(\bar{G}_{3}-G_{3}\right) \wedge \bar{\omega}_{A_{1}} \int \bar{G}_{3} \wedge \bar{\omega}_{A_{2}}\right\} .
\end{aligned}
$$

\subsection{Calculating the mass terms for D7-branes with 2-form fluxes}

We are interested exclusively in the 2 -form fluxes corresponding to the angles of intersecting branes in the $T$-dual picture.

When the 2-form fluxes, discussed in the previous section are turned on, the Kähler potential and therefore also the metric and $H$-term receive a flux dependence. The metric and $H$-term for the $D 7$-brane position modulus $C_{3}^{7}$ take the following form: 


$$
G_{C_{3}^{7} \bar{C}_{3}^{7}}=\frac{-1}{(U-\bar{U})(S-\bar{S})}+\frac{\alpha^{\prime-2} f_{12} f_{34}}{(T-\bar{T})(U-\bar{U})}, \quad H=-G .
$$

With this, we get the following curvature tensor components:

$$
\begin{aligned}
R_{U \bar{U} 3 \overline{3}} & =\frac{-1}{(U-\bar{U})^{2}} G_{C_{3}^{7} \bar{C}_{3}^{7}}, \\
R_{S \bar{S} 3 \overline{3}} & =\frac{2 \alpha^{\prime-2} f_{12} f_{34}(S-\bar{S})-(T-\bar{T})}{(S-\bar{S})^{3}(U-\bar{U})\left[\alpha^{\prime-2} f_{12} f_{34}(S-\bar{S})-(T-\bar{T})\right]}, \\
R_{T \bar{T} 3 \overline{3}} & =\frac{\alpha^{-2} f_{12} f_{34}\left[2(T-\bar{T})-\alpha^{\prime-2} f_{12} f_{34}(S-\bar{S})\right]}{(T-\bar{T})^{3}(U-\bar{U})\left[\alpha^{\prime-2} f_{12} f_{34}(S-\bar{S})-(T-\bar{T})\right]}, \\
R_{T \bar{S} 3 \overline{3}} & =\frac{-\alpha^{\prime-2} f_{12} f_{34}}{(S-\bar{S})(T-\bar{T})(U-\bar{U})\left[\alpha^{\prime-2} f_{12} f_{34}(S-\bar{S})-(T-\bar{T})\right]} .
\end{aligned}
$$

This results in a changed soft scalar mass term:

$$
\begin{aligned}
m_{C^{7 a} \bar{C}^{7 a}, \text { soft }}^{2}= & \left.Y\right|^{-1}\left\{\frac{-1}{(S-\bar{S})(U-\bar{U})}\left[\left|\int G_{3} \wedge \Omega\right|^{2}+\left|\int G_{3} \wedge \omega_{A_{1}}\right|^{2}+\left|\int G_{3} \wedge \omega_{A_{2}}\right|^{2}\right]\right. \\
& +\frac{\alpha^{\prime-2} f_{12} f_{34}}{(U-\bar{U})\left[\alpha^{\prime-2} f_{12} f_{34}(S-\bar{S})-(T-\bar{T})\right]}\left[\left|\int G_{3} \wedge \Omega\right|^{2}+\left|\int G_{3} \wedge \omega_{A_{1}}\right|^{2}\right. \\
& \left.+\left|\int G_{3} \wedge \omega_{A_{2}}\right|^{2}+2\left|\int \bar{G}_{3} \wedge \Omega\right|^{2}-\left(\int G_{3} \wedge \Omega \int G_{3} \wedge \bar{\Omega}+\text { h.c. }\right)\right] \\
& -\frac{\left(\alpha^{\prime-2} f_{12} f_{34}\right)^{2}(S-\bar{S})}{(U-\bar{U})(T-\bar{T})\left[\alpha^{\prime-2} f_{12} f_{34}(S-\bar{S})-(T-\bar{T})\right]}\left[\left|\int \bar{G}_{3} \wedge \Omega\right|^{2}\right. \\
& \left.\left.+\left|\int G_{3} \wedge \omega_{A_{1}}\right|^{2}+\left|\int G_{3} \wedge \omega_{A_{2}}\right|^{2}\right]\right\}
\end{aligned}
$$

Turning on 2-form flux has therefore led to the contribution of more 3-form flux components to the mass terms than before, as had been realized already in [20]. The limit $f_{12}, f_{34} \rightarrow \infty$ changes the boundary conditions from Neumann to Dirichlet such that the $D 7$-brane is converted into a $D 3$-brane. In this limit the piece quadratic in the two-form fluxes is leading and describes precisely the soft-breaking mass terms on the D3-brane [39,40].

The $\mu$-term is calculated as before, but with the modified $H$-term (6.25). This results in:

$$
\mu_{C^{7} C^{7}}=Y^{-1 / 2}\left[\frac{-1}{(S-\bar{S})(U-\bar{U})} \int \bar{G}_{3} \wedge \omega_{A_{3}}+\frac{\alpha^{\prime-2} f_{12} f_{34}}{(T-\bar{T})(U-\bar{U})} \int G_{3} \wedge \omega_{A_{3}}\right] .
$$


In the limit $f_{12}, f_{34} \rightarrow \infty$ we again recover the $D 3$-brane result of only $(1,2)$-form fluxes contributing to $\mu$ [39,40]. The contribution of the $\mu$-term to the total mass term is $G^{C^{7} \bar{C}^{7}}|\mu|^{2}$ :

$$
\begin{aligned}
G^{C^{7} \bar{C}^{7}\left|\mu_{C^{7} C^{7}}\right|^{2}=} & |Y|^{-1}\left\{\frac{-1}{(S-\bar{S})(U-\bar{U})}\left|\int \bar{G}_{3} \wedge \omega_{A_{3}}\right|^{2}\right. \\
& +\frac{\alpha^{\prime-2} f_{12} f_{34}}{(U-\bar{U})\left[-\alpha^{\prime-2} f_{12} f_{34}(S-\bar{S})-(T-\bar{T})\right]}\left[-\left|\int \bar{G}_{3} \wedge \omega_{A_{3}}\right|^{2}\right. \\
& \left.+\left(\int \bar{G}_{3} \wedge \omega_{A_{3}} \int \bar{G}_{3} \wedge \bar{\omega}_{A_{3}}+\text { h.c. }\right)\right] \\
& \left.+\frac{\left(\alpha^{\prime-2} f_{12} f_{34}\right)^{2}(S-\bar{S})}{(U-\bar{U})(T-\bar{T})\left[\alpha^{\prime-2} f_{12} f_{34}(S-\bar{S})-(T-\bar{T})\right]}\left|\int G_{3} \wedge \omega_{A_{3}}\right|^{2}\right\} .
\end{aligned}
$$

Notice that not the entire contribution of the $\mu$-term originates from $(2,1)$-form fluxes anymore. Only the terms

$$
\begin{aligned}
& |Y|^{-1}\left\{\frac{-1}{(S-\bar{S})(U-\bar{U})}\left|\int \bar{G}_{3} \wedge \omega_{A_{3}}\right|^{2}\right. \\
& \left.-\frac{\alpha^{\prime-2} f_{12} f_{34}}{(U-\bar{U})\left[-\alpha^{\prime-2} f_{12} f_{34}(S-\bar{S})-(T-\bar{T})\right]}\left|\int \bar{G}_{3} \wedge \omega_{A_{3}}\right|^{2}\right\}
\end{aligned}
$$

proportional to $\left|\int \bar{G}_{3} \wedge \omega_{A_{3}}\right|^{2}$ preserve supersymmetry. The total mass term is $m_{\text {total }}^{2}=m_{\text {soft }}^{2}+G^{C^{7} \bar{C}^{7}}|\mu|^{2}$.

To conclude, we have derived the soft susy-breaking mass terms and an effective $\mu$-term, which depends on $(2,1)$-form fluxes only in the case of vanishing worldvolume 2 -form flux on the $D 7$-branes. In the presence of world-volume 2 -form fluxes, the effective $\mu$-term receives a $(1,2)$-dependent piece. On the other hand, the masses $m_{C^{7} \bar{C}^{7}}$ of scalar fields of $D 7$-brane moduli, describing their positions, receive both $3-$ and $2-$ form flux dependent contributions.

\section{Conclusions}

In this paper we have discussed the superpotential and the vacuum structure of F-theory on $K 3 \times K 3$ with general 4-form flux turn on. In string theory this corresponds to type $I I B$ on $K 3 \times T^{2}$ with non-constant dilaton, with non-vanishing 3 -form flux in the closed (bulk) string sector and with $D 7$-branes equipped with non-zero open string 2-form flux. As a result of our discussion it turns out that 
for generic fluxes all open and closed string moduli are fixed, except for a volume factor 22 Furthermore the minimization of the open string $D 7$-brane moduli is such that the orientifold is generically unstable, i.e. that the positions of the $D 7$-branes are different from their orientifold limits (and that the dilaton is non-constant in a generic F-theory flux vacuum). However we also showed that there exist particular choices of F-theory fluxes which drive the theory to a stable orientifold limit. We also compare these results with an effective supergravity formulation of the flux compactification in terms of $\mathcal{N}=2$ gauged supergravity.

This last observation is important for the second part of the paper where we discuss the $\mu$-term and the softly supersymmetry breaking terms on the $D 7$-brane world-volume in the orientifold limit, and in particular in the orbifold limit of $K 3$. The result of this discussion is that the effective supersymmetric mass terms of nonchiral fermions of the $D 7$-brane moduli fields depend both on $(2,1)$-form fluxes as well as on (1,2)-form fluxes in case of non-vanishing 2-form fluxes on the $D 7$-branes. In addition, we should emphasize that these results should be used in the following way: the derived equations for the $\mu$-term and the soft terms are parametrized by the values of the moduli fields $(S, T, U)$ and also by the values of the 2-form and 3 -form fluxes. However in any vacuum the moduli are generically fixed and hence are functions of a given choice of 2-form and 3-form fluxes. Therefore, in order to derive actual (numerical) values for the soft terms, one first has to determine the vacuum in any given flux compactification, i.e. the fixed values for moduli, and use them as input in the equations for the $\mu$-term and the soft terms. Of course there might exist special combinations of soft term (certain ratios etc) which are essentially moduli independent and therefore do not require the full determination of the non-supersymetric vacuum structure.

Acknowledgements: We are grateful to H. Jockers and J. Louis for valuable discussions and informing us about their related work prior to publication. We also thank M. Trigiante for valuable discussions. This work is supported in part by the Deutsche Forschungsgemeinschaft as well as by the EU-RTN network Constituents, Fundamental Forces and Symmetries of the Universe (MRTN-CT-2004-005104). S.R. thanks the university of Munich for hospitality.

22 Note, that this statement includes also the twisted sector of orbifold limits. Generalized flux compactifications, where different types of moduli can be fixed to various degrees have been recently discussed also in other setups, in particular for all but a single volume modulus in [36], for all geometric bulk moduli in [41,42], and for a subset of orbifold moduli in [43]. 


\section{Appendix A. Some general facts on K3}

In the following we collect some facts on the cohomology $H^{*}(X)$ of a $\mathrm{K} 3 X$ and period integrals defined on it; we refer to 444 45 for more background material. The integral cohomology $H^{\text {even }}(X)$ has the structure of a selfdual lattice $\Gamma^{4,20}$ of signature -16 . Splitting off a factor $H^{0} \oplus H^{4}$ of signature $(1,1)$, the orthogonal sublattice $\Gamma^{3,19} \subset \Gamma^{4,20}$ corresponds to the 2 -form cohomology, generated by 3 selfdual and 19 anti-selfdual 2-forms. Fixing an embedding $H^{2}(X, \mathbf{Z}) \rightarrow \Gamma^{3,19}$ and choosing a basis $\left\{\eta_{I}\right\}$ for $H^{2}(X, \mathbf{Z})$, the inner product on $\Gamma^{3,19}$ is defined by

$$
M_{I J}=\int \eta_{I} \wedge \eta_{J}=\left(\begin{array}{ccccc}
U & & & & \\
& U & & & \\
& & U & & \\
& & -C_{E 8} & \\
& & & -C_{E 8}
\end{array}\right), \quad U=\left(\begin{array}{ll}
0 & 1 \\
1 & 0
\end{array}\right),
$$

where $-C_{E_{8}}$ denotes the negative of the Cartan matrix for $E_{8}$. An Einstein metric at volume one is specified, up to discrete identifications, by choosing a space-like 3-plane in $\mathbf{R}^{3,19} \supset \Gamma^{3,19}$ spanned by the 3 orthogonal, selfdual forms $\omega^{i}, i=1, . ., 3$. Taking into account discrete identifications corresponding to isometries of $\Gamma^{3,19}$, the moduli space is

$$
O\left(\Gamma^{3,19}\right) \backslash O(3,19) / O(3) \times O(19) \times \mathbf{R}_{+},
$$

where the last factor parametrizes the volume.

Fixing a direction in the $S U(2)$ of complex structures, the $\omega^{i}$ combine into a holomorphic 2-form, say $\omega=\omega^{1}+i \omega^{2}$ and the Kähler form $j=\omega^{3}$ as in (2.3); they fulfill

$$
\omega \wedge \omega=j \wedge \omega=0, \quad \int j \wedge j>0, \quad \int \omega \wedge \bar{\omega}>0 .
$$

These 2-forms may be expanded as $\omega=\Pi^{I} \eta_{I}, j=\Pi^{3, I} \eta_{I}$, with expansion coefficients given by the period integrals

$$
\Pi^{I}=\int_{\gamma_{I}} \omega=\int \omega \wedge \eta^{I}, \quad \Pi^{3, I}=\int_{\gamma_{I}} j,
$$

where $\left\{\gamma_{I}\right\}$ denote the basis of $H_{2}(X, \mathbf{Z})$ dual to the $\left\{\eta_{I}\right\}$ and indices are raised and lowered with the help of the metric (A.1) and its inverse.

From the first equation in (A.3), the periods $\Pi^{I}$ satisfy the quadratic constraint

$$
\Pi^{I} M_{I J} \Pi^{J}=0 .
$$


The independent periods may serve as local projective coordinates on the moduli space of complex structures.

The Picard group of $X$ is defined as the group of integral $(1,1)$ forms

$$
\operatorname{Pic}(X)=H^{2}(X, \mathbf{Z}) \cap H^{1,1}(X),
$$

which implies $\int \omega \wedge \eta_{I}=0$ for $\eta_{I} \in \operatorname{Pic}(X)$. For generic complex structure, $\operatorname{Pic}(X)$ is trivial. However for the elliptic fibered $\widetilde{X}_{V}$ with section, the duals of the elliptic fiber $E$ and the dual of the section $B$ are elements of $\operatorname{Pic}(X)$ with intersections (2.6). The complex structure is determined by the period vector $\Pi^{I}$ on the remaining 20 basis elements. For the following it is sometimes convenient to use an orthogonal basis for the lattice, with intersection matrix

$$
M_{I J}=\operatorname{diag}\left(2,2,-2,-2,-1^{16}\right) .
$$

In this basis the period vector reads

$$
\Pi_{I}=\left(\begin{array}{c}
1-S U+\frac{1}{2} C^{a} C^{a} \\
S+U \\
1+S U-\frac{1}{2} C^{a} C^{a} \\
-S+U \\
-C^{a}
\end{array}\right)
$$

where we have fixed the projective action in the patch where $\Pi_{1}+\Pi_{3} \neq 0$ and we have also used the constraint (A.4).

The period vector (A.7) describes a set of standard coordinates on the coset $S O(2,18) /(S O(2) \times S O(18))$. Note that in this parametrization an $S O(2,2 ; \mathbf{Z})$ of reparametrizations is realized linearly on the periods $S$ and $U$. The same period vector also appears as the upper half of a special choice for the symplectic section of $\mathcal{N}=2$ supergravity based on the special Kähler manifold (4.5). In particular it has been proposed in [24] that this section describes the special geometry for the type IIB orientifold on $T^{2} / \mathbf{Z}_{2}$. However despite of the similarity, the period vector (A.7) at $C^{a}=0$ cannot be identified with the symplectic section for the type IIB orientifold on $T^{2} / \mathbf{Z}_{2}$. This follows e.g. from the uniqueness of the selfdual lattice with signature $(2,18)$ which implies that the vanishing cycles at $C^{a}=0$ must span the root lattice of $E_{8} \times E_{8}$. The proper symplectic section is described by a transformation from (A.7) to a different basis that is described in app. B below. 


\section{Appendix B. Orientifold limit $X^{\sharp}$ of $\widetilde{X}_{V}$}

The non-abelian gauge symmetry of the orientifold limit of type IIB on $T^{2} / \mathbf{Z}_{2}$ is $S O(8)^{4}$ from $4 \mathrm{D} 7$-branes on top of each of the orientifold planes. To describe the corresponding singular K3 $X^{\sharp}$, we need a parametrization different from (A.7), such that the vanishing cycles correspond to four copies of root lattice of $D_{4}$. Note that the orientifold limit is also not described by the simple orbifold $T^{2} \times T^{2} / \mathbf{Z}_{2}$, whose vanishing cohomology has intersection matrix $A_{1}^{16}$, but by resolving three $A_{1}$ singularities in a different orbifold denoted by $T^{4} / \widehat{D}_{4}^{\prime}$. 3 . The description of the generators of the integral cohomology of K3 orbifolds in terms of the vanishing cohomology and the cohomology inherited from $T^{4}$ is quite delicate. We refer to 46] for a complete discussion of the cohomology of K3 orbifolds without translations as well as for references to the mathematical literature on the subject.

In the following we restrict ourselves to describe a parametrization of the period vector in an appropriate basis for the orientifold which does however not generate $H^{2}\left(X^{\sharp}, \mathbf{Z}\right)$ over the integers. The integrality properties may be recovered from transforming integral fluxes in the basis corresponding to (A.7) to the new basis described below.

To find the appropriate basis change, we may borrow from the results on the dual heterotic string compactified on $T^{2}$ with Wilson lines [47] 10. In the orthogonal basis with intersection matrix (A.6), the roots of a single $E_{8}$ factor correspond to the 240 vectors of the form

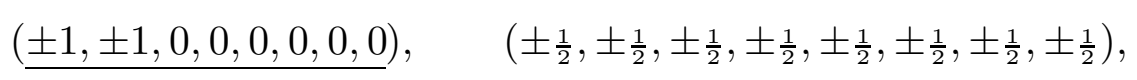

with all possible choices of signs for the l.h.s. and an even number of minus sign for the r.h.s. These roots become orthogonal to $\omega$ in the limit $C^{a}=0, a=1, \ldots, 8$ in (A.7). Similarly a different copy of $E_{8}$ roots becomes orthogonal to $\omega$ if in addition $C^{a}=0, a=9, \ldots, 16$. In the following the two $E_{8}$ factors enter symmetrically.

To describe the limit with roots in $4 D_{4}$ instead, we first change the complex structure from $C^{a}=0$ to

$$
v=\left(1,0,0,0, \frac{U}{2}, \frac{U}{2}, \frac{U}{2}, \frac{U}{2}\right) .
$$

23 Strictly speaking these comments refer to the manifold $\widetilde{X}_{V}$ that appears in the M-theory compactification to 7 dimensions, before taking the F-theory limit. 
It is readily verified that the roots orthogonal to $\omega$ span two factors of the $D_{4}$ root lattice for any $U$. Including the second $E_{8}$ factor and after change of basis and shifting the coordinates one arrives at a new "period vector"

$$
\Pi_{I}=\left(\begin{array}{c}
1-S U+\frac{1}{2} C^{a} C^{a} \\
S+U \\
-1-S U-\frac{1}{2} C^{a} C^{a} \\
-S+U \\
-C^{a}
\end{array}\right)
$$

Let us stress that although the period vector looks formally identical to (A.7), it is not so for two reasons. Firstly the locus $C^{a}=0$ corresponds to a vanishing cohomology given by the root lattice $4 D_{4}$. Secondly the basis vectors do not span the integral lattice over the integers. See [46] for an appropriate basis of generators for the integral lattice in terms of exceptional divisors and invariant forms. However one may also reconstruct the integral vectors from the known lattice vectors in the basis (A.7) and the known transformation from (A.7) to (B.3). One may also verify explicitely that there is a choice of integral flux that drives the theory to the orientifold limit above, or also the orbifold $T^{4} / \mathbf{Z}_{2}$ discussed in the text.

\section{Appendix C. Three-form fluxes of $T^{4} / \mathbf{Z}_{2} \times T^{2}$}

In this appendix we present the (untwisted) three-form cohomology, used before to describe the three-form flux $G_{3}$ of the type $I I B$ orientifold compactification $T^{4} / \mathbf{Z}_{2} \times T^{2}$. For toroidal orbifolds $T^{6} / \mathbf{Z}_{N}$ the complex structures are introduced through:

$$
d z^{j}=\sum_{i=1}^{3} \rho_{i}^{j} d x^{i}+\tau_{i}^{j} d y^{i}, \quad j=1,2,3 .
$$

For the case of the direct product $T^{6}=T^{4} / \mathbf{Z}_{2} \times T^{2}$ we may choose $\rho_{i}^{j}=\delta_{i}^{j}$ and $\tau_{i}^{3}=\delta_{i}^{3} U^{3}$. In addition, for the present case, the $T^{4}$ being itself the direct product of two two-tori $T^{4}=T^{2,1} \times T^{2,2}$, we have $\tau_{i}^{j}=\delta_{i}^{j} U^{j}$. To this end, we have: $d z^{i}=d x^{i}+U^{i} d y^{i}, i=1,2,3$. 
The 3-forms of $T^{4} / \mathbf{Z}_{2} \times T^{2}$ are obtained by wedging the one-forms $d z^{3}$ or $d \bar{z}^{3}$ of the torus $T^{2,3}$ to the 2 -forms given in (5.48), which means that we end up with twelve 3 -forms from the untwisted sector:

$$
\begin{array}{ll}
\omega_{A_{0}}=d \bar{z}^{1} \wedge d \bar{z}^{2} \wedge d \bar{z}^{3} \quad, \quad \omega_{B_{0}}=d z^{1} \wedge d z^{2} \wedge d z^{3}, \\
\omega_{A_{1}}=d \bar{z}^{1} \wedge d z^{2} \wedge d z^{3}, & \omega_{B_{1}}=d z^{1} \wedge d \bar{z}^{2} \wedge d \bar{z}^{3}, \\
\omega_{A_{2}}=d z^{1} \wedge d \bar{z}^{2} \wedge d z^{3} & , \quad \omega_{B_{2}}=d \bar{z}^{1} \wedge d z^{2} \wedge d \bar{z}^{3}, \\
\omega_{A_{3}}=d z^{1} \wedge d z^{2} \wedge d \bar{z}^{3} & , \quad \omega_{B_{3}}=d \bar{z}^{1} \wedge d \bar{z}^{2} \wedge d z^{3} \\
\omega_{A_{4}}=d z^{1} \wedge d \bar{z}^{1} \wedge d z^{3} & , \quad \omega_{B_{4}}=d \bar{z}^{1} \wedge d z^{1} \wedge d \bar{z}^{3}, \\
\omega_{A_{5}}=d z^{2} \wedge d \bar{z}^{2} \wedge d z^{3} & , \quad \omega_{B_{5}}=d \bar{z}^{2} \wedge d z^{2} \wedge d \bar{z}^{3} .
\end{array}
$$

The forms $\omega_{A_{0}}, \ldots, \omega_{A_{3}}$ and $\omega_{B_{0}}, \ldots, \omega_{B_{3}}$ fulfill the primitivity condition $J \wedge \omega=0$, with the Kähler form $J=\sum_{j=1}^{3} \operatorname{Im}\left(\mathcal{T}^{j}\right) d z^{j} \wedge d \bar{z}^{j}$. On the other hand, the forms $\omega_{A_{4}}, \omega_{A_{5}}, \omega_{B_{4}}, \omega_{B_{5}}$ are not primitive, i.e. $J \wedge \omega \neq 0$, yet it is possible to form two primitive linear combinations from them:

$$
\omega_{A_{p}}=\omega_{A_{5}}-\frac{\operatorname{Im}\left(\mathcal{T}^{1}\right)}{\operatorname{Im}\left(\mathcal{T}^{2}\right)} \omega_{A_{4}}, \quad \omega_{B_{p}}=\omega_{B_{5}}-\frac{\operatorname{Im}\left(\mathcal{T}^{1}\right)}{\operatorname{Im}\left(\mathcal{T}^{2}\right)} \omega_{B_{4}} .
$$

In $E q$. (5.42) the flux $G_{3}$ is expanded w.r.t. to the real cohomology:

$$
\begin{array}{ll}
\alpha_{0}=d x^{1} \wedge d x^{2} \wedge d x^{3} \quad, \quad \beta^{0}=d y^{1} \wedge d y^{2} \wedge d y^{3} \\
\alpha_{1}=d y^{1} \wedge d x^{2} \wedge d x^{3} \\
\alpha_{2}=d x^{1} \wedge d y^{2} \wedge d x^{3} \quad, \quad \beta^{1}=-d x^{1} \wedge d y^{2} \wedge d y^{3} \\
\alpha_{3}=d x^{1} \wedge d x^{2} \wedge d y^{3}, \quad, \quad \beta^{3}=-d y^{1} \wedge d x^{2} \wedge d y^{3} \\
\alpha_{4}=d x^{1} \wedge d y^{1} \wedge d x^{3} \wedge d x^{3} \\
\alpha_{5}=d x^{2} \wedge d y^{2} \wedge d x^{3}, \quad, \quad \beta^{4}=-d x^{1} \wedge d y^{1} \wedge d y^{3}
\end{array}
$$

In this basis, the forms $\alpha_{4}, \alpha_{5}, \beta^{4}$ and $\beta^{5}$ are non-primitive. At any rate, three-form flux components w.r.t. the latter do not give a contribution in the superpotential (5.43). The $(3,0)$-form may be expanded w.r.t. the basis (C.4):

$$
\begin{aligned}
\Omega & =\alpha_{0}+U^{1} \alpha_{1}+U^{2} \alpha_{2}+U^{3} \alpha_{3} \\
& -U^{2} U^{3} \beta^{1}-U^{1} U^{3} \beta^{2}-U^{1} U^{2} \beta^{3}+U^{1} U^{2} U^{3} \beta^{0} .
\end{aligned}
$$




\section{References}

[1] S. Gukov, C. Vafa and E. Witten, "CFT's from Calabi-Yau four-folds," Nucl. Phys. B 584, 69 (2000) [Erratum-ibid. B 608, 477 (2001)] arXiv:hep-th/9906070].

[2] K. Dasgupta, G. Rajesh and S. Sethi, "M theory, orientifolds and G-flux," JHEP 9908, 023 (1999) arXiv:hep-th/9908088.

[3] S. B. Giddings, S. Kachru and J. Polchinski, "Hierarchies from fluxes in string compactifications," Phys. Rev. D 66, 106006 (2002) arXiv:hep-th/0105097.

[4] T. R. Taylor and C. Vafa, "RR flux on Calabi-Yau and partial supersymmetry breaking," Phys. Lett. B 474, 130 (2000) arXiv:hep-th/9912152.

[5] P. Mayr, "On supersymmetry breaking in string theory and its realization in brane worlds," Nucl. Phys. B 593, 99 (2001) arXiv:hep-th/0003198.

[6] C. Vafa, "Evidence for F-Theory," Nucl. Phys. B 469, 403 (1996) arXiv:hepth/9602022].

[7] K. Becker and M. Becker, "M-Theory on Eight-Manifolds," Nucl. Phys. B 477, 155 (1996) arXiv:hep-th/9605053.

[8] M. Haack and J. Louis, "M-theory compactified on Calabi-Yau fourfolds with background flux," Phys. Lett. B 507, 296 (2001) arXiv:hep-th/0103068.

[9] D. R. Morrison and C. Vafa, "Compactifications of F-Theory on Calabi-Yau Threefolds - I,II," Nucl. Phys. B 476, 437 (1996) arXiv:hep-th/9603161]; Nucl. Phys. B 473, 74 (1996) arXiv:hep-th/9602114.

[10] L. Görlich, S. Kachru, P. K. Tripathy and S. P. Trivedi, "Gaugino condensation and nonperturbative superpotentials in flux compactifications," arXiv:hep-th/0407130.

[11] A. Sen, "F-theory and Orientifolds," Nucl. Phys. B 475, 562 (1996) arXiv:hepth/9605150].

[12] S. Sethi, C. Vafa and E. Witten, "Constraints on low-dimensional string compactifications," Nucl. Phys. B 480, 213 (1996) arXiv:hep-th/9606122.

[13] P. K. Tripathy and S. P. Trivedi, "Compactification with flux on K3 and tori," JHEP 0303, 028 (2003) arXiv:hep-th/0301139.

[14] M. Aganagic and C. Vafa, "Mirror symmetry, D-branes and counting holomorphic discs," arXiv:hep-th/0012041.

[15] E. Witten, "Chern-Simons gauge theory as a string theory," Prog. Math. 133, 637 (1995) arXiv:hep-th/9207094.

[16] H. Jockers and J. Louis, to appear. 
[17] W. Lerche, P. Mayr and N. Warner, "Holomorphic N = 1 special geometry of openclosed type II strings," arXiv:hep-th/0207259; W. Lerche and P. Mayr, "On N = 1 mirror symmetry for open type II strings," arXiv:hep-th/0111113.

[18] D. Lüst, "Intersecting brane worlds: A path to the standard model?," Class. Quant. Grav. 21, S1399 (2004) arXiv:hep-th/0401156; R. Blumenhagen, "Recent progress in intersecting D-brane models," arXiv:hep-th/0412025.

[19] S. Ferrara, C. Kounnas and M. Porrati, "General Dimensional Reduction Of TenDimensional Supergravity And Superstring," Phys. Lett. B 181, 263 (1986).

[20] D. Lüst, S. Reffert and S. Stieberger, "Flux-induced soft supersymmetry breaking in chiral type IIb orientifolds with D3/D7-branes," arXiv:hep-th/0406092.

[21] P.G. Camara, L.E. Ibanez and A.M. Uranga, "Flux-induced SUSY-breaking soft terms on D7-D3 brane systems," arXiv:hep-th/0408036.

[22] D. Lüst, S. Reffert and S. Stieberger, "MSSM with soft SUSY breaking terms from D7-branes with fluxes," arXiv:hep-th/0410074;

A. Font and L.E. Ibanez, "SUSY-breaking soft terms in a MSSM magnetized D7-brane model," arXiv:hep-th/0412150.

[23] L. Andrianopoli, R. D'Auria, S. Ferrara and M. A. Lledo, "4-D gauged supergravity analysis of type IIB vacua on $K 3 \times T^{2} / \mathbf{Z}_{2}$," JHEP 0303, 044 (2003) arXiv:hepth/0302174].

[24] C. Angelantonj, R. D'Auria, S. Ferrara and M. Trigiante, " $K 3 \times T^{2} / \mathbf{Z}_{2}$ orientifolds with fluxes, open string moduli and critical points," Phys. Lett. B 583, 331 (2004) arXiv:hep-th/0312019.

[25] B. de Wit, V. Kaplunovsky, J. Louis and D. Lüst, "Perturbative couplings of vector multiplets in N=2 heterotic string vacua," Nucl. Phys. B 451, 53 (1995) arXiv:hepth/9504006];

I. Antoniadis, S. Ferrara, E. Gava, K. S. Narain and T. R. Taylor, "Perturbative prepotential and monodromies in $\mathrm{N}=2$ heterotic superstring," Nucl. Phys. B 447, 35 (1995) arXiv:hep-th/9504034.

[26] L. Andrianopoli et al., " $\mathrm{N}=2$ supergravity and $\mathrm{N}=2$ super Yang-Mills theory on general scalar manifolds: Symplectic covariance, gaugings and the momentum map," J. Geom. Phys. 23, 111 (1997) arXiv:hep-th/9605032.

[27] G. Curio, A. Klemm, D. Lüst and S. Theisen, "On the vacuum structure of type II string compactifications on Calabi-Yau spaces with H-fluxes," Nucl. Phys. B 609, 3 (2001) arXiv:hep-th/0012213. 
[28] J. Michelson, "Compactifications of type IIB strings to four dimensions with nontrivial classical potential," Nucl. Phys. B 495, 127 (1997) arXiv:hep-th/9610151;

G. Dall'Agata, "Type IIB supergravity compactified on a Calabi-Yau manifold with H-fluxes," JHEP 0111, 005 (2001) arXiv:hep-th/0107264.

[29] S. Cecotti, L. Girardello and M. Porrati, "Two Into One Won’t Go," Phys. Lett. B 145, 61 (1984).

[30] S. Ferrara, L. Girardello and M. Porrati, "Minimal Higgs Branch for the Breaking of Half of the Supersymmetries in N=2 Supergravity," Phys. Lett. B 366, 155 (1996) arXiv:hep-th/9510074.

[31] I. Antoniadis, C. Bachas, C. Fabre, H. Partouche and T.R. Taylor, "Aspects of type I - type II - heterotic triality in four dimensions," Nucl. Phys. B 489, 160 (1997) arXiv:hep-th/9608012.

[32] H. Jockers and J. Louis, "The effective action of D7-branes in N = 1 Calabi-Yau orientifolds," Nucl. Phys. B 705, 167 (2005) arXiv:hep-th/0409098.

[33] D. Lüst, P. Mayr, R. Richter and S. Stieberger, "Scattering of gauge, matter, and moduli fields from intersecting branes," Nucl. Phys. B 696, 205 (2004) arXiv:hepth/0404134.

[34] S. Stieberger, " $(0,2)$ heterotic gauge couplings and their M-theory origin," Nucl. Phys. B 541, 109 (1999) arXiv:hep-th/9807124.

[35] F. Ardalan, H. Arfaei and M. M. Sheikh-Jabbari, "Noncommutative geometry from strings and branes," JHEP 9902, 016 (1999) arXiv:hep-th/9810072.

[36] I. Antoniadis and T. Maillard, "Moduli stabilization from magnetic fluxes in type I string theory," arXiv:hep-th/0412008.

[37] L.E. Ibanez and D. Lüst, "Duality anomaly cancellation, minimal string unification and the effective low-energy Lagrangian of 4-D strings," Nucl. Phys. B 382, 305 (1992) arXiv:hep-th/9202046;

V.S. Kaplunovsky and J. Louis, "Model independent analysis of soft terms in effective supergravity and in string theory," Phys. Lett. B 306, 269 (1993) arXiv:hepth/9303040].

[38] G.F. Giudice and A. Masiero, "A Natural Solution To The Mu Problem In Supergravity Theories," Phys. Lett. B 206, 480 (1988).

[39] P.G. Camara, L.E. Ibanez and A.M. Uranga, "Flux-induced SUSY-breaking soft terms," Nucl. Phys. B 689, 195 (2004) arXiv:hep-th/0311241. 
[40] M. Grana, T.W. Grimm, H. Jockers and J. Louis, "Soft supersymmetry breaking in Calabi-Yau orientifolds with D-branes and fluxes," Nucl. Phys. B 690, 21 (2004) arXiv:hep-th/0312232.

[41] S. Kachru and A.K. Kashani-Poor, "Moduli potentials in type IIA compactifications with RR and NS flux," arXiv:hep-th/0411279.

[42] T. W. Grimm and J. Louis, "The effective action of type IIA Calabi-Yau orientifolds," arXiv:hep-th/0412277.

[43] J.P. Derendinger, C. Kounnas, P.M. Petropoulos and F. Zwirner, "Superpotentials in IIA compactifications with general fluxes," arXiv:hep-th/0411276.

[44] P. S. Aspinwall, "K3 surfaces and string duality," arXiv:hep-th/9611137.

[45] D.R. Morrison, "Some remarks on the moduli of K3 surfaces", Progress in Math., vol. 39, Birkhäuser, 1983, pp. 303-332.

[46] K. Wendland, "Consistency of orbifold conformal field theories on K3," Adv. Theor. Math. Phys. 5, 429 (2002) arXiv:hep-th/0010281.

[47] J. Polchinski, "String theory, Vol. 2", Cambridge University Press, 1998. 